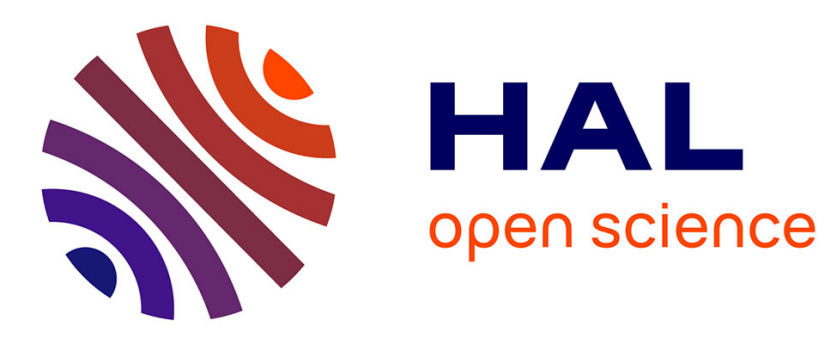

\title{
Image processing for precise three-dimensional registration and stitching of thick high-resolution laser-scanning microscopy image stacks
}

\author{
Chloé Murtin, Carole Frindel, David Rousseau, Kei Ito
}

\section{To cite this version:}

Chloé Murtin, Carole Frindel, David Rousseau, Kei Ito. Image processing for precise three-dimensional registration and stitching of thick high-resolution laser-scanning microscopy image stacks. Computers in Biology and Medicine, 2018, 92, pp.22 - 41. 10.1016/j.compbiomed.2017.10.027 . hal-01690908

\section{HAL Id: hal-01690908 https://hal.science/hal-01690908}

Submitted on 23 Jan 2018

HAL is a multi-disciplinary open access archive for the deposit and dissemination of scientific research documents, whether they are published or not. The documents may come from teaching and research institutions in France or abroad, or from public or private research centers.
L'archive ouverte pluridisciplinaire HAL, est destinée au dépôt et à la diffusion de documents scientifiques de niveau recherche, publiés ou non, émanant des établissements d'enseignement et de recherche français ou étrangers, des laboratoires publics ou privés. 


\title{
Image processing for precise three-dimensional registration and stitching of thick high-resolution laser-scanning microscopy image stacks
}

\author{
Chloé Murtin ${ }^{\mathrm{a}, \mathrm{b}, \mathrm{c}}$, Carole Frindel ${ }^{\mathrm{c}}$, David Rousseau ${ }^{\mathrm{c}, \mathrm{e}, *}$, Kei Ito ${ }^{\mathrm{a}, \mathrm{b}, \mathrm{d}}$ \\ ${ }^{a}$ Institute of Molecular and Cellular Biosciences, The University of Tokyo, Yayoi, Bunkyo-ku, 113-0032 Tokyo, Japan \\ ${ }^{\mathrm{b}}$ Department of Computational Biology, Graduate School of Frontier Sciences, The University of Tokyo, Kashiwanoha, Kashiwa, 277-0882 Chiba, Japan \\ ${ }^{\mathrm{c}}$ CREATIS, Institut National des Sciences Appliquées de Lyon (INSA Lyon), 7 Avenue J Capelle, bat. Blaise Pascal, F-69621 Villeurbanne cedex, France \\ ${ }^{\mathrm{d}}$ Institut für Zoologie, Universität zu Köln, Zülpicher Str. 47b, 50674 Köln, Germany \\ e LARIS, UMR IRHS INRA, 62 Avenue Notre Dame du Lac, Université d'Angers, France
}

\section{A R T I C L E I N F O}

\section{Keywords:}

Stitching

Registration

SIFT

Laser-scanning microscopy

Brain

Connectomics

Drosophila

\begin{abstract}
A B S T R A C T
The possible depth of imaging of laser-scanning microscopy is limited not only by the working distances of objective lenses but also by image degradation caused by attenuation and diffraction of light passing through the specimen. To tackle this problem, one can either flip the sample to record images from both sides of the specimen or consecutively cut off shallow parts of the sample after taking serial images of certain thickness. Multiple image substacks acquired in these ways should be combined afterwards to generate a single stack. However, subtle movements of samples during image acquisition cause mismatch not only in the translation along x-, $\mathrm{y}$-, and $\mathrm{z}$-axes and rotation around $\mathrm{z}$-axis but also tilting around $\mathrm{x}$ - and $\mathrm{y}$-axes, making it difficult to register the substacks precisely. In this work, we developed a novel approach called 2D-SIFT-in-3D-Space using Scale Invariant Feature Transform (SIFT) to achieve robust three-dimensional matching of image substacks. Our method registers the substacks by separately fixing translation and rotation along x-, y-, and z-axes, through extraction and matching of stable features across two-dimensional sections of the 3D stacks. To validate the quality of registration, we developed a simulator of laser-scanning microscopy images to generate a virtual stack in which noise levels and rotation angles are controlled with known parameters. We illustrate quantitatively the performance of our approach by registering an entire brain of Drosophila melanogaster consisting of 800 sections. Our approach is also demonstrated to be extendable to other types of data that share large dimensions and need of fine registration of multiple image substacks. This method is implemented in Java and distributed as ImageJ/Fiji plugin. The source code is available via Github (http://www.creatis.insa-lyon.fr/site7/fr/MicroTools).
\end{abstract}

\section{Introduction}

Laser-scanning fluorescent microscopy is a powerful tool for analyzing three-dimensional (3D) complex structures found in life sciences such as neuronal structures, which can be visualized using fluorophore-conjugated antibody labeling or targeted-expression of fluorescent proteins [1]. Combinations of multiple fluorescent markers and excitation filters can be used to highlight various neuronal objects, e.g., axons as well as pre- and postsynaptic sites, in the same specimen [2]. Such imaging techniques promise to give access, for instance in neurology, to connectomics which aims to produce a comprehensive and systematic analysis of the connections between brain regions and between numerous neurons within them [3]. The success of such analysis depends on the capacity to acquire in great detail, i.e., at the scale of the synapsis, the entire volume of the brain specimen.

Although laser-scanning microscopy can acquire images of thin optical sections from thick tissues [4], the possible depth of imaging is limited by three factors. First, the working distance of the microscope objective limits the depth for which images can be recorded. Second, signals become darker in deeper regions of the samples, because both excitation laser beam and emitted fluorescence are attenuated by the sample tissue that is optically not completely translucent [5]. This causes diminution of signal intensity and signal-to-noise ratio. And third, image quality degrades in deeper regions even when it is still within the working distance of the lens. Although new techniques such as clearing agents (e.g., CLARITY and Scale) can make sample tissues transparent [6, 7], light rays are deflected and scattered when they pass through tissues. Deep objects therefore appear blurry and lose contrast.

\footnotetext{
* Corresponding author. LARIS, UMR IRHS INRA, 62 Avenue Notre Dame du Lac, Université d'Angers, France.

E-mail address: david.rousseau@univ-angers.fr (D. Rousseau).
} 
Thus, spatial resolution decreases with the depth from the sample surface. Even with the objectives with high numerical aperture and long working distance (more than $200 \mu \mathrm{m}$ ), image quality decreases considerably when the focal plane becomes as deep as $100 \mu \mathrm{m}$ [8]. Two work around techniques have been employed to overcome this problem. For the specimen that is thinner than $300 \mu \mathrm{m}$ along the optical axis (z-axis), it is possible to record the image from both sides of the sample that is embedded between thin cover slips. The first image substack covering the frontal half of the sample is taken from one side of the cover slip. The sample is then flipped, and the second image substack is recorded from the other side. For a thicker specimen, the sample can be embedded in a soft medium and using tissue sectioning (e.g., vibratome), a sample section is cut off from the top of the sample block after taking image stacks of this section. By repeating this procedure, called two-photon tomography, in principle any thick specimen can be imaged $[9,10]$.

In both approaches, image substacks should be acquired in an overlapping manner: the overlapping sections will serve as a guide indicating how neighboring substacks can be concatenated, or stitched. However, such concatenation is not straightforward, because small rotation and translation can occur when the sample is flipped or when the block surface is cut off. Rotation can occur not only around the z-axis of the specimen but also around $x$ - and y-axes (tilting). Moreover, because of the photobleaching that occurs during image stack acquisition and because of the different depths from the sample surface, the intensity of the corresponding optical sections in two overlapping stacks often appear different.

This is why registration is crucial for properly stitching image substacks of the same sample. Each neuronal fiber from neighboring stacks must be perfectly connected to each other after stitching. A discontinuity in the final image stack would strongly affect further analysis such as neuron tracing $[2,11,12]$. Registration is one of the most important general problems of image processing [13]. Therefore, there exists a wide offer of commercial or free, manual or automated, 2D or 3D, software platforms that address this issue. Application specific solutions, however, that are relevant for common types of samples and acquisition protocols, are often difficult to access for life scientist. Such a perspective could be judged as relatively narrow in a general computer vision context. However, as recently illustrated in this journal [14-17], it is actually specifically meaningful in biomedical imaging where important communities of life scientists work on the same types of samples.

In this study, we have developed an optimized automatic registration and stitching algorithm, 2D-SIFT-in-3D-Space, specifically adapted for thick high-resolution laser-scanning microscopy image stacks. A visual flow chart of the proposed algorithm is given in Fig. 1. We applied our method to stitch large 3D image stacks of Drosophila melanogaster brain samples that can be mutually tilted by up to $20^{\circ}$. Our algorithm, made available under the open source Fiji software that is widely used through the international bioimaging community, combines several existing approaches into a new strategy based on reliably detecting features in images using scale invariant feature transform (SIFT). In addition, to validate quantitatively the registration quality, we developed an original simulator that generates artificial 3D image stacks that mimic the properties of noise in laser scanning microscopy. We have used 2D-SIFT-in3D-Space algorithm to assemble 3D image stacks of neurons of the Drosophila brain at a voxel resolution of $0.2 \times 0.2 \times 0.2 \mu \mathrm{m}$ $(1600 \times 1600$ voxels and 800 sections). The stitched dataset serves as a starting point for characterizing fine architecture of such large entire brain at unprecedented resolution but the 2D-SIFT-in-3D-Space is also shown to be useful to other types of datasets and other fluorescent microscopy systems (see supplementary data).

\section{Related work}

The primary purpose of the proposed registration method, 2D-SIFTin-3D-Space, is to stitch two image substacks. The registration is achieved by comparing the signals of two overlapping image stacks. In this context,

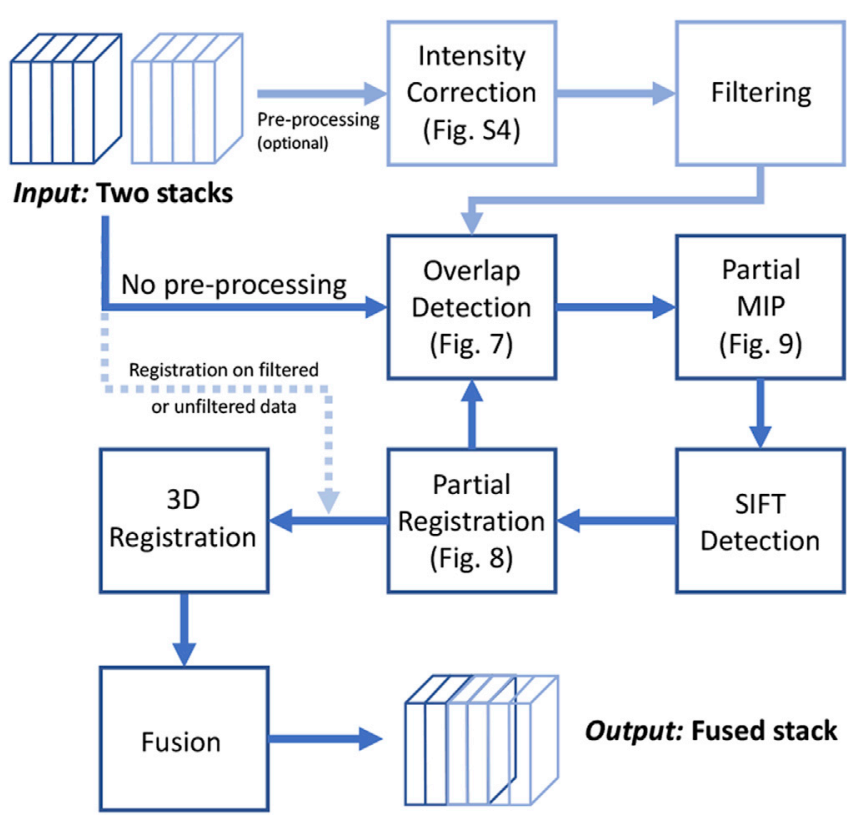

Fig. 1. Flow chart of the proposed automatic registration and stiching algorithm 2D-SIFTin-3D Space.

it shares some similarities with existing registration algorithms known to be adapted to the bioimaging community interested in the registration of 3D images. A possible classification for image registration approaches is whether the registration is based on intensity or on features [18].

Intensity-based approaches confront, with a correlation metric, the intensity patterns in images to be registered. This includes for instance software solutions such as CMTK (Computational Morphometry Toolkit), elastiX, ANTS, AMIRA. Intensity-based approaches are specifically suited when the homologous structures to be registered in the 3D stacks are well represented by the intensities through the spatial statistics of the gray levels seen as a random variable (see for instance in spatial intensity [19], or with Fourier transform of the intensities [20], or also as recently used in Ref. [21]).

Feature-based approaches, as chosen and developed in this article, realize a correspondence between homologous landmarks in the images to be registered. Feature-based approaches are specifically suited when the images are characterized by spurious small structures (e.g., vessels and blobs) such as the one highlighted in the samples of neuronal fiber images considered in this article. Feature-based approaches works in two steps: First the detection of landmarks and second the match of the homologous landmarks in the images. In some software solutions the landmarks have to be detected with another software or selected manually. Manual positioning of such landmarks can be very time consuming and can also be perturbed by human errors. To circumvent this difficulty automatic detection of homologous landmarks is preferred. This can be achieved by detecting fiducial structures of known shape and size such as fluorescence beads that are purposely added to the sample [22] or endogenous granules in the tissue [23]. Registration error, however, would occur if samples and added beads were put in fluid mounting medium such as glycerol used in our study. Whereas [23] utilized endogenous aging-related pigment granules that are distributed across the mouse neural tissue, similar approach was not applicable to our samples because such granules do not seem to be distributed ubiquitously in young fly brains. Automatic detection of homologous landmarks can also be done purely numerically based on the extraction of local image features (See Ref. [24] for a review). The great advantage of feature-based registration is that instead of using all image intensities, it is possible to register two stacks using only corresponding salient points as a statistic of the image content. Reducing the problem from full resolution image content to a relatively small number of corresponding 

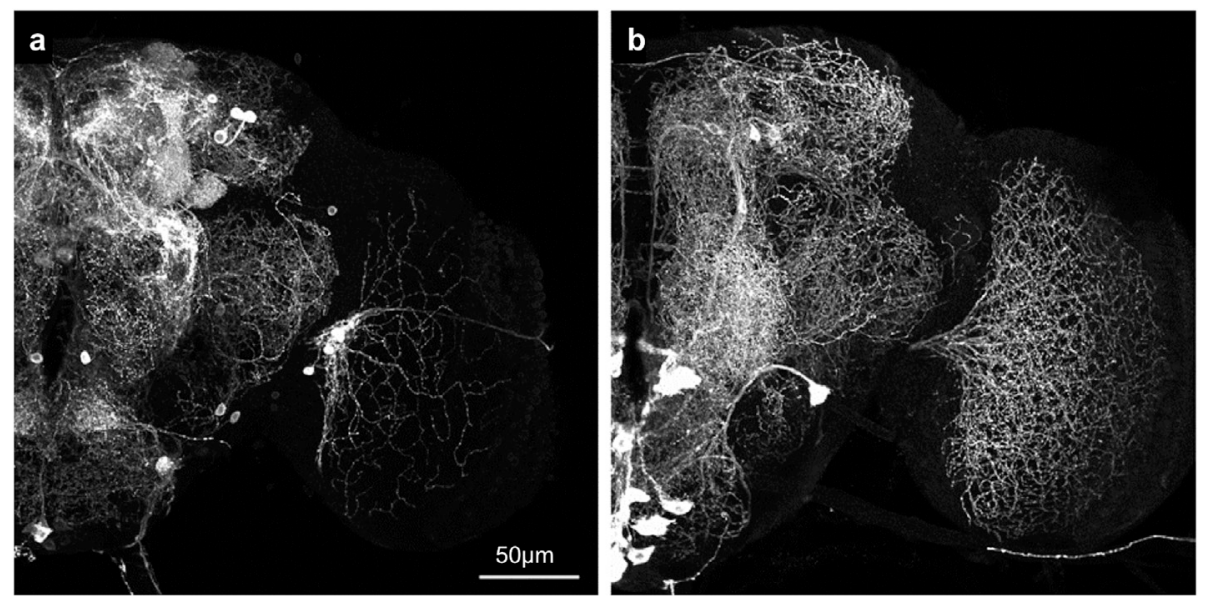

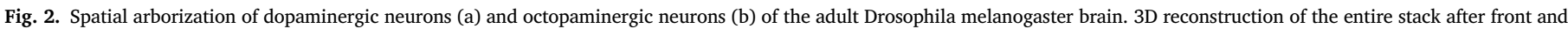
back substacks are registered and merged. $1600 \times 1600 \times 800$ voxels, voxel resolution $=0.2 \times 0.2 \times 0.2 \mu \mathrm{m}$. Scale bar $=50 \mu \mathrm{m}$.

homologous points simplifies the estimation of the transformation model and is a major computing time saver.

Feature-based and intensity-based techniques have been shown to be useful for registering image stacks of different tissue samples as well as for stitching sections and image substacks of a single tissue. Software designed for the stitching purpose tends to assume that all the sections are parallel without tilting (e.g., [20]) and do not correct rotation mismatches around all axes (see Supplementary data for a comparison with our solution). Software designed for the registration purpose can generally correct rotation and translation in all 3D axes, but many of them are designed for registering image stacks that cover the same part of the specimen in different samples. To operate on the type of data considered in this article, one would require two image processing steps: first the manual crop of the overlapping part and second the stitching of the entire registered stacks. Effective manual crop may appear trivial for the samples with small tilting angles, but is actually a difficult task for large angles when dealing with self-similar samples such as the neurons in the brain. The existing techniques are, to the best of our knowledge, not natively capable of joint automatic detection of the overlap and registration and stitching of 3D image substacks that overlap only partially as found with the thick samples imaged with high-resolution laser-scanning microscopy.

To stitch image substacks with tilting errors, we propose a solution with an original use of a popular local feature SIFT introduced by Ref. [25]. Whereas we achieved 3D registration with the repeated use of 2D SIFT in three orthogonal planes, SIFT in true 3D space has also been proposed for motion recognition of video images [26], object recognition for X-ray computer tomography images [27], or in biomedical applications [28-31]. Although the same approach can in principle be applied to develop an image registration software, the choice of 2D-SIFT-in-3D-Space is well adapted in our case, because the specific image acquisition sequence considered in this article stitching of microscope image substacks obtained from the same sample induces more important rotations around z-axis than the tilting around other axes; the first iteration of registration in $x-y$ plane is thus very likely to bring a strong improvement. This prior would not be used with 3D SIFT registration, which would blindly look for solutions in any direction of the 3D space.

\section{Materials and methods}

\subsection{Definition of the biological task}

As a model system, we used the brain of the adult fruit fly Drosophila melanogaster. It contains about 100,000 neurons in the volume that is approximately $600-\mu \mathrm{m}$ wide, $300-\mu \mathrm{m}$ tall, and $160-\mu \mathrm{m}$ thick. The fly brain has been an intense focus for brain-wide analysis of neural networks and their functions $[3,32,33]$. Projection patterns of neuronal fibers and distribution of synaptic connection sites can be visualized by expressing proteins that are spread along cytoplasm and those that are transported to presynaptic sites [32]. A wide variety of neuron types can be visualized using cell-specific expression driver strains [1]. Among them the dopaminergic and octopaminergic neurons - which are known to be involved in diverse brain functions - form extensive projections in almost all the brain regions [34,35]. Because those neurons feature complex arborizations that are much denser and finer than most other neuron types (Fig. 2), high-resolution microscopy images are required. To this aim, images of the fluorescent antibody-labeled samples were recorded using confocal laser-scanning microscopes (Olympus FV1000 and FV1200) with a 40x silicon immersion objective $(\mathrm{NA}=1.25)$ at an image resolution of $0.2 \times 0.2 \times 0.2 \mu \mathrm{m}$ each, in total $1600 \times 1600$ pixels and approximately 800 serial sections.

Image quality degrades as the plane of the scanning optical section goes deeper into the specimen (Fig. 3) even with high-resolution objective lenses that are designed to match the refraction index of the mounting medium. Thus, although the total thickness of the samples (Ca. $160 \mu \mathrm{m}$ ) is well within the working distance of the objective lens (Ca. $280 \mu \mathrm{m}$ ), deeper half of the samples cannot be recorded with optimum resolution if they are imaged only from one side. To address this issue, we mounted the specimen between thin cover slips on both sides with a space of $200-\mu \mathrm{m}$ thickness, and the deeper half of the sample is recorded from the other side by flipping the preparation (Fig. 4). The two substacks, taken from the front and back sides of the brain, were then merged after flipping the volume data of the back substack.

This approach works well if the samples are mounted in rigid medium and if the sample after flipping can be placed exactly at $180^{\circ}$ from the original direction. However, fluorescent samples are often mounted in a fluid substrate such as $80 \%$ glycerol, in which the specimen are not completely fixed but stay afloat. Slight rotation may therefore occur when the samples are flipped. In addition, because of the instrumental error, the two cover slips may not completely be in parallel, causing the flip not to be exactly at $180^{\circ}$. Such error would not cause severe problems if the images were taken at relatively low resolution. However, to reconstruct fine neuronal fibers which are often thinner than $0.5 \mu \mathrm{m}$, even subtle misalignment results in discontinuity such as gaps, if the substacks are concatenated without fine three-dimensional registration (Fig. 5).

\subsection{D-SIFT-in-3D-space algorithm}

Our registration and stitching algorithm consists of several steps 

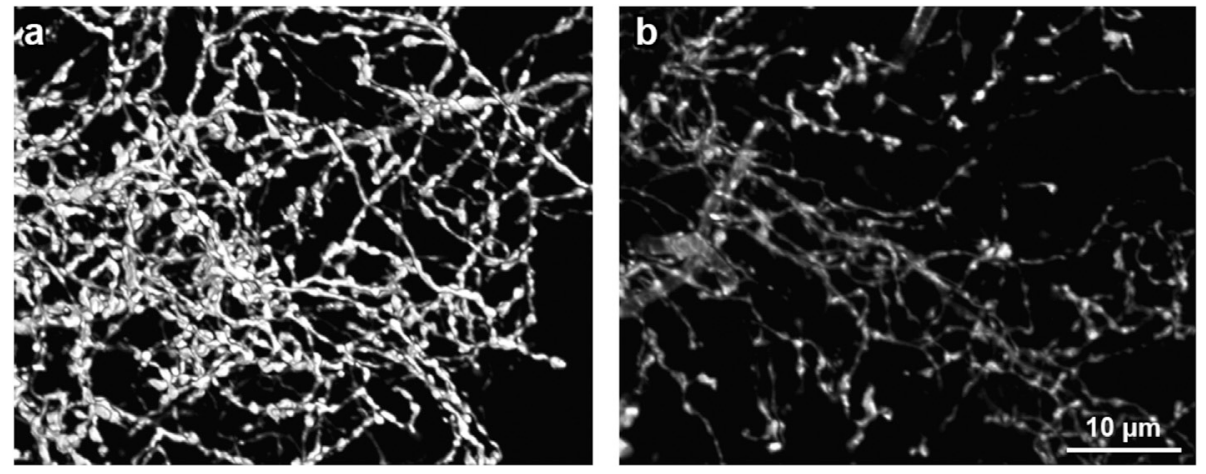

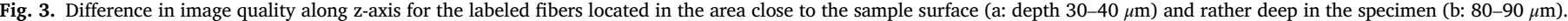
Note that not only brightness but also sharpness is decreased. Scale bar $=10 \mu \mathrm{m}$.
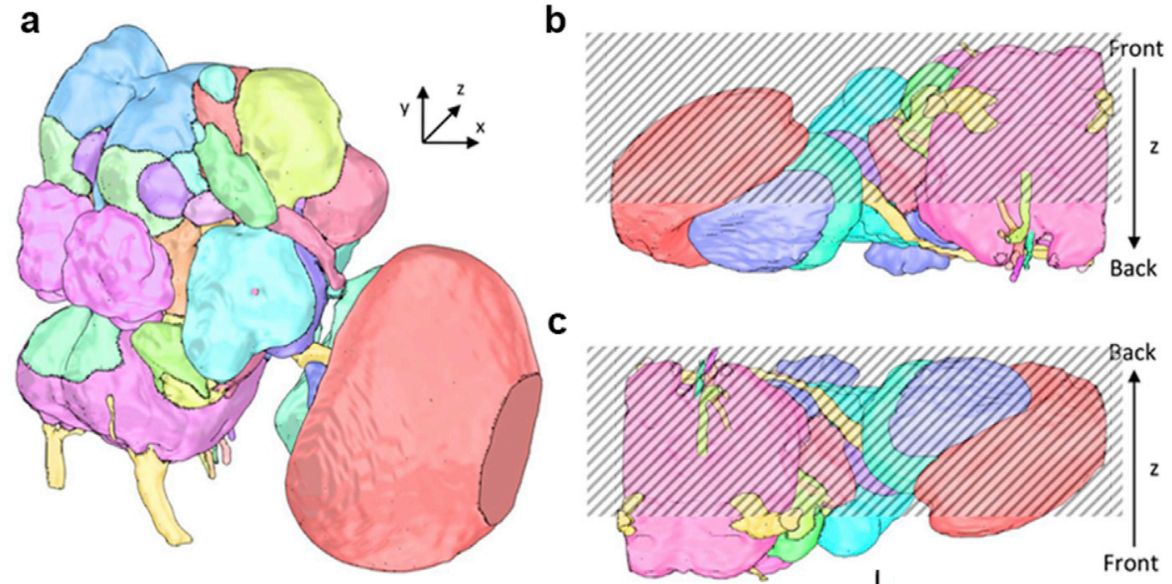

d

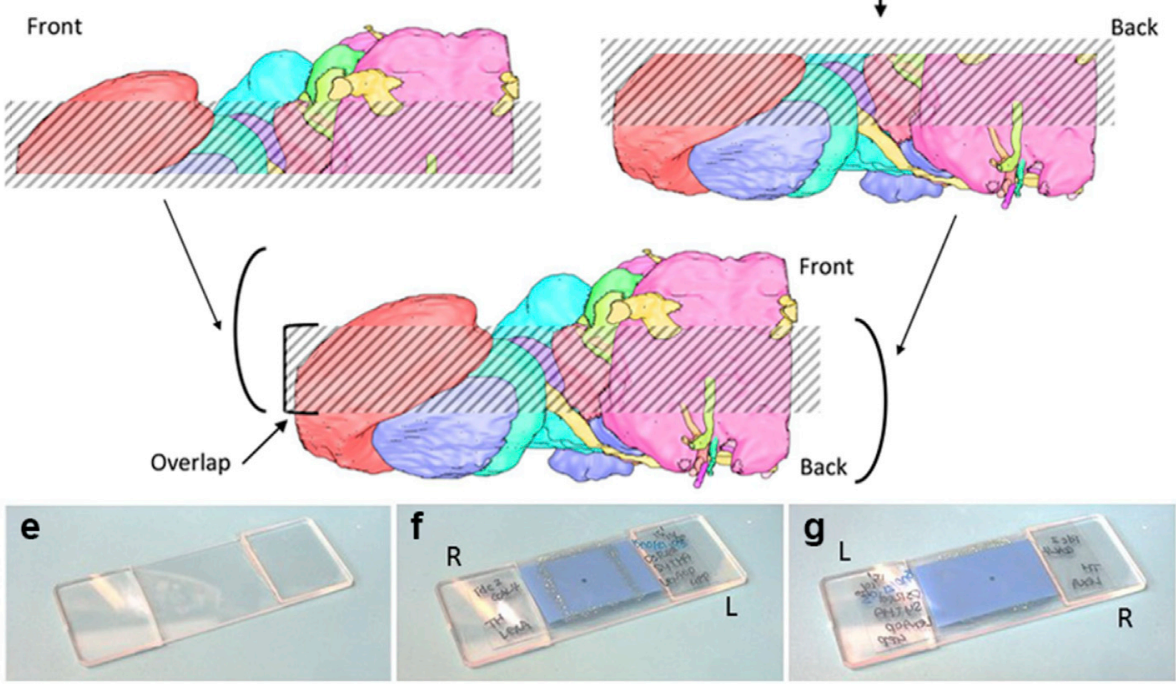



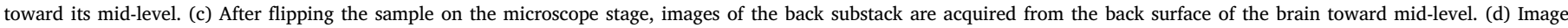



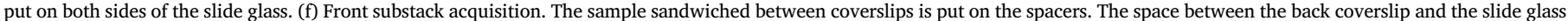
avoids the occurrence of Newton ring that occurs when two glass material contact each other. $(\mathrm{g}$ ) The sample is flipped for back substack acquisition.

(Fig. 6): (1) the overlap detection in the two data substacks, (2) partial maximum intensity projection (MIP), (3) filter selection, (4) SIFT features extraction and correspondence detection, (5) affine transformation parameters estimation and (6) registered overlapping portions fusion. The details of all the steps will be described in the following sections.
Overlap detection. This step aims to find the portions of the two image substacks containing overlapping image data. To do so, we compare the image content across sections of the two substacks using SIFT features (see Fig. 7a1-2). For each section comparison, the number of corresponding SIFT features is stored which allows the computation of the 

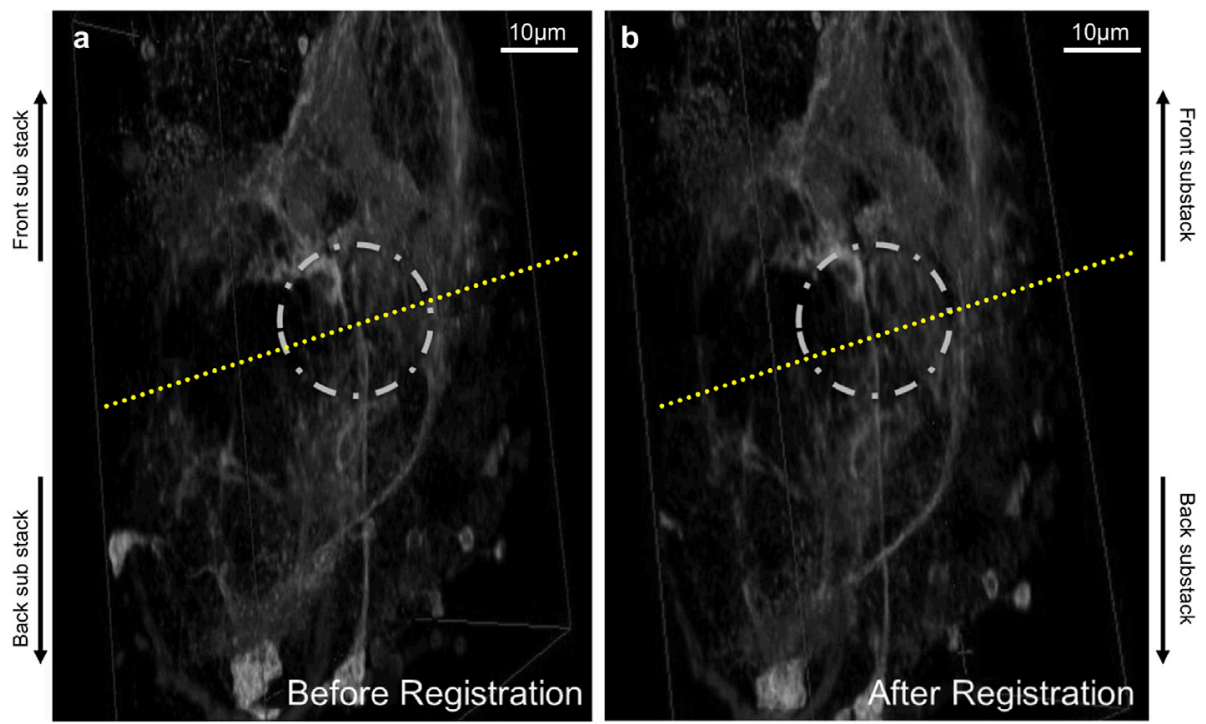

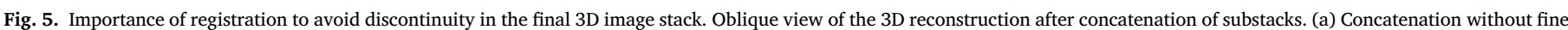

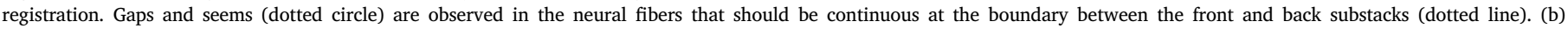
Concatenation after fine registration. Fibers appear continuous.

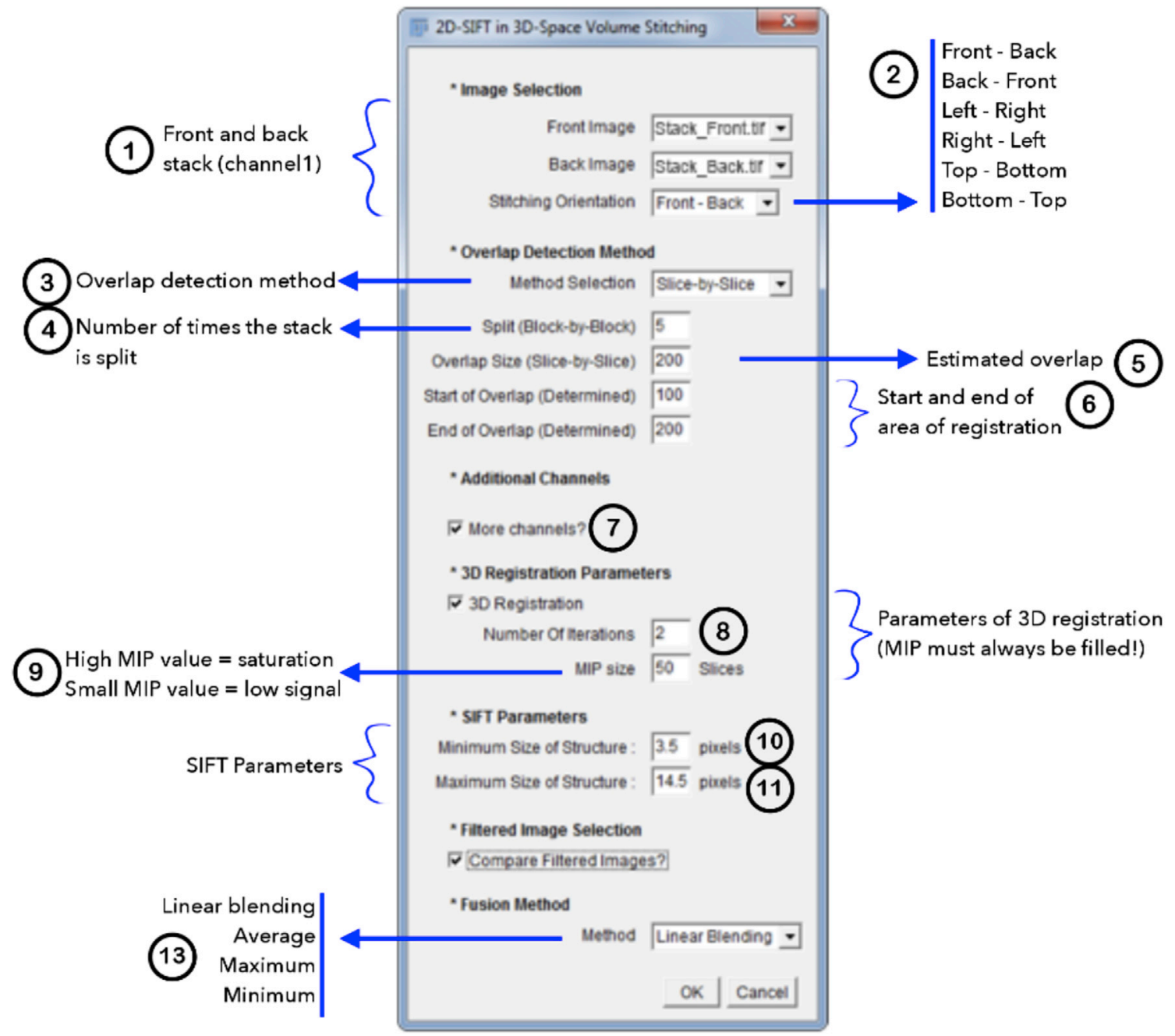

Fig. 6. Control panel of the 2D-SIFT-in-3D-space Volume Stitching plugin, implemented for ImageJ/Fiji. See main text for detail. 


\section{a Slice-by-slice comparison}

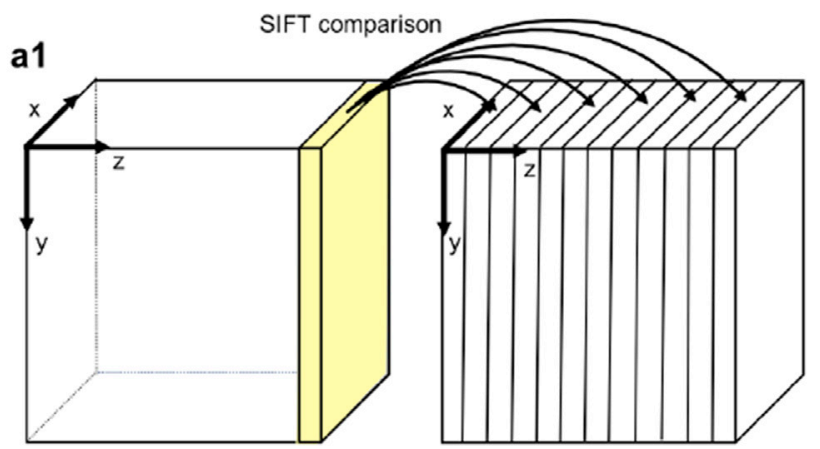

Front substack

Back substack a2

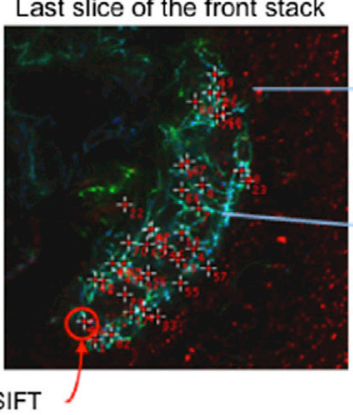

Slice from the back stack

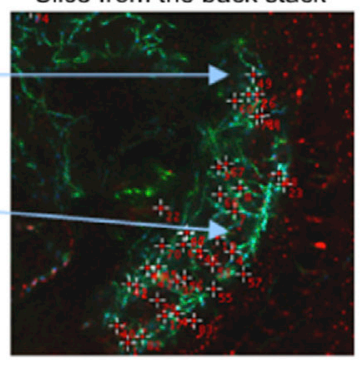

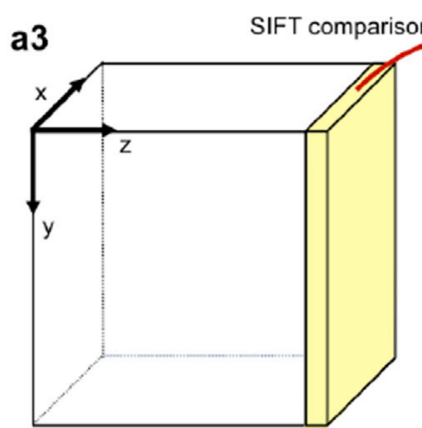

Front substack
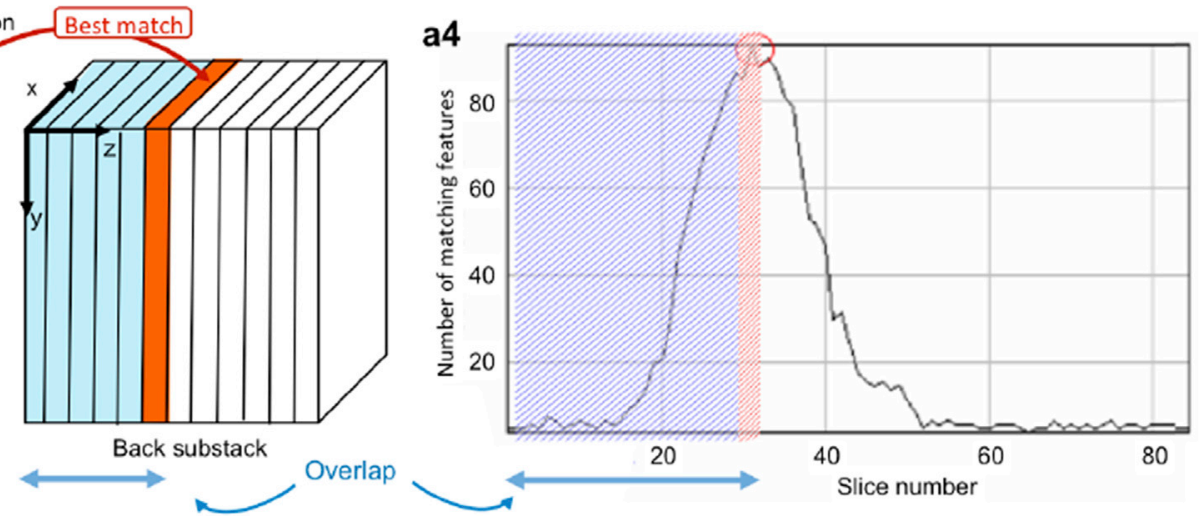

\section{b Block-by-block comparison}

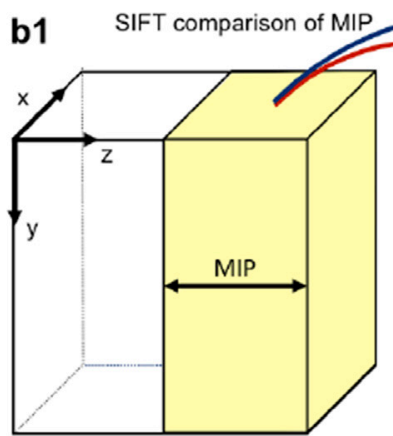

Front substack

a3

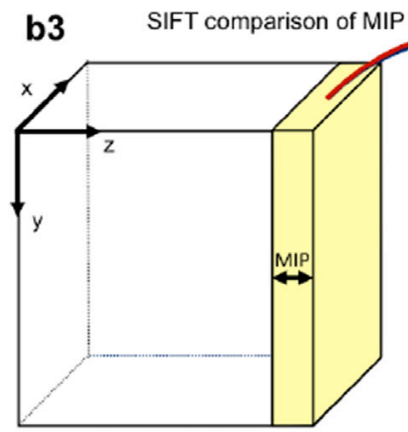

Front substack

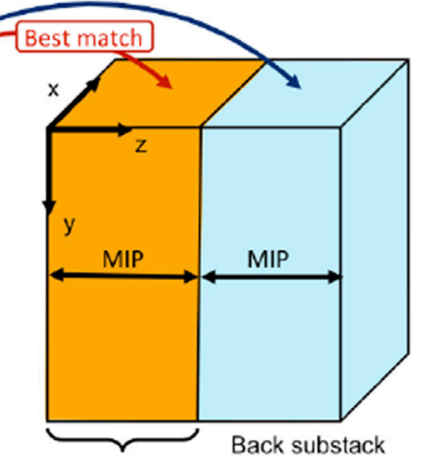

Used for further splitting



Front substack

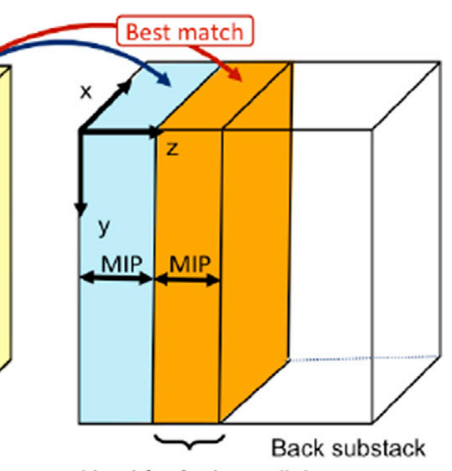

Used for further splitting

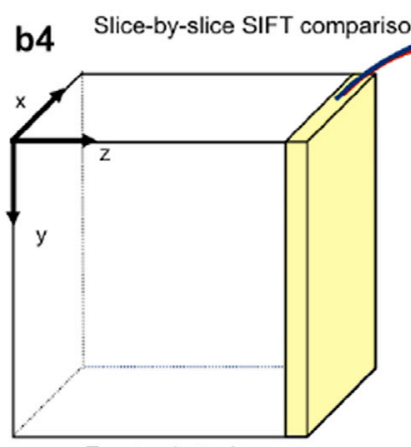

Front substack

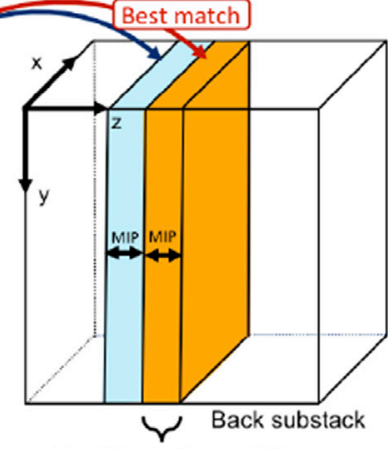

Used for further splitting

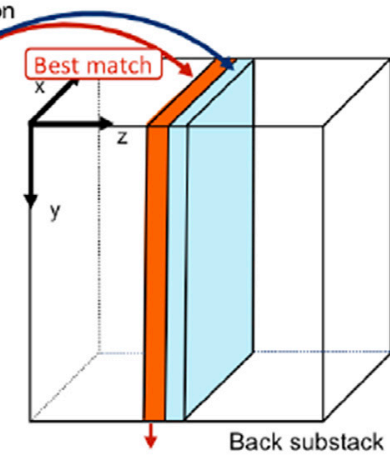

Overlap position

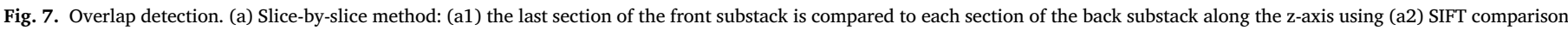

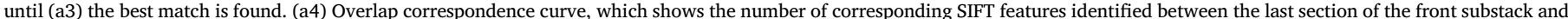

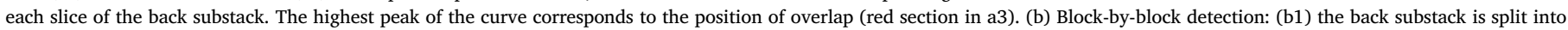

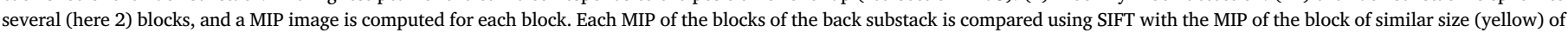


correspondence curve where the overlap positions are detected with the highest peak of the correspondence curve (see Fig. 7a3-4). To detect the overlap, we proposed two alternative approaches. The first approach performs a slice-by-slice comparison: the last section of the front substack is compared to each section of the back substack along the z-axis (Fig. 7a). The overlap positions are detected with the highest peak of the correspondence curve, in other words the section of the back substack with most common information with the last section of the front substack. This approach is based on the assumption that tilting between the planes of the two substacks is usually small enough to find important similarities between substack sections of the corresponding depths within the overlap. However, slice-by-slice comparison requires long computation time on substacks made of numerous sections. Moreover, when the tilting between the two substacks is big, similarity between sections is often not sufficient for identifying corresponding sections. For those reasons, we developed an alternative approach, block-by-block comparison (Fig. 7b) using partial MIP presented in the following section.

Partial MIP. In this approach, we split the two entire substacks into several blocks and make the maximum intensity projection of the sections within each block (partial MIP). SIFT comparison is then performed between the partial MIP of the last block of the front substack with the partial MIP of each block in the back substack (Fig. 7b1). The number of blocks is determined by the parameter Split set in the control panel (Fig. 6). For example, if the splitting parameter is set at 2, the substacks will be divided into two blocks. The partial MIP of the last block of the front substack will then be compared with the MIPs of the two backsubstack blocks and the best match is selected. The selected backsubstack block is further split in two incrementally and compared with the equally split last block of the front substack (Fig. 7b2-3), until the successively split blocks contain only one section (Fig. 7b4). The splitting parameter can be set by the user, and the effect of its choice will be discussed in the results section.

Filter selection. This step allows using preprocessed (filtered) images instead of original stack images to calculate registration parameters. The transformation model will be computed by comparing the SIFT features of the filtered images, but the resulting model will be applied to the original data. Applying various filters to enhance biological structures to be registered may boost feature extraction and thus improve the final stitching on real images. For example, for our specific application, one can use a vesselness filter to enhance tubular structures of neuronal fiber images. For more generic applications, one can choose local contrast filter to enhance faint signals or denoising filter to eliminate misleading signals. Such filters are available in ImageJ/Fiji software. Another effective way of using preprocessed images is to perform registration in 8-bit images instead of original images with larger bit depth (e.g., 12-bit or 16-bit), which will significantly reduce computation time. However, stitching of such preprocessed images may not be scientifically pertinent, because it may affect signal intensity and distribution as well as bit-depth resolution. To provide users full flexibility, final concatenation can be performed either with the preprocessed data or unprocessed raw image stacks.

SIFT features extraction and correspondence detection. Comparison of sections of the two substacks is performed using SIFT [25]. SIFT is a local descriptor that allow both automatic identification of salient points in a section - by detecting blobs within a specified size range using the Difference of Gaussian detector [36] - and extraction of features for these points. A feature consists in an invariant descriptor to scaling, orientation, and partially invariant to affine distortion and illumination changes. For this step, we use the version of 2D SIFT algorithm developed by Stephan Saalfeld (http://fiji.sc/Feature_Extraction). Following this implementation, we provide two key parameters: (i) minimum and (ii) maximum size of biological structures (in pixels) to be detected, as depicted in Fig. 6. SIFT features are extracted at all scales between maximum and minimum size. Correspondence between SIFT features is identified by nearest neighbor matching in the local descriptor space. However, it results in a significant number of false correspondences. In our registration context, the set of SIFT correspondences are related by an unknown 2-d affine transformation $T$. The tool we used to separate true and false correspondences is the random sample consensus (RANSAC) implementation proposed by Refs. [37,38]. In short, it works as follows: for a fixed number of iterations, it randomly selects a set of correspondence candidates and estimates $T$ for them. The residual error of all candidates in terms of $T$ is calculated and candidates with a residual error lower than some maximum displacement are collected as true correspondences. The largest set of true correspondences found is used to estimate the optimal $T$. Number of iterations and maximal displacement error are parameters of the implementation and were left to default values proposed in the implementation of $[37,38]$.

Affine transformation parameters estimation in three planes. Registration is performed using only the data of the overlapping portions (Fig. 8a). Three successive rounds of registration are performed in three orthogonal planes (Fig. 8b-d), where SIFT comparison is performed between spatially corresponding pairs of slices. First, the overlapping data portions of the front and back substacks are re-sliced along the z-axis to obtain cross-section slices in the $x-y$ plane (Fig. 8b). Slices of similar depth are compared using SIFT features between front and back overlapping portions to calculate translation parameter in the $x-y$ plane and rotation parameter around z-axis. Second, the re-slicing step is performed along $\mathrm{x}$-axis to obtain cross sections in the $\mathrm{y}$-z plane, and the generated slices are compared to calculate translation in the $y-z$ plane and rotation around $\mathrm{x}$-axis (Fig. 8c). Finally, the re-slicing step is performed along yaxis to calculate in the same way translation in the $\mathrm{x}-\mathrm{z}$ plane and rotation around y-axis (Fig. 8d). This combination of steps allows for a 3D registration starting with the plane containing the most information and the potentially more displacement (which is linked to the acquisition protocol which rotates the sample according to the $x$-y plane). In theory, iterative uses of 2D SIFT might increase the chance that the whole registration would fail. It is not the case in our approach, because we initiate the iterative process in the direction of the most likely important transformation.

In this processing step, comparison of single sections within the overlapping portion often do not contain enough amount of corresponding signals, so that extracted SIFT features in the section of the front substack may match with only a tiny number of features in a section of the back substack. To overcome this problem, instead of comparing single sections, we again proposed the use of partial MIP (Fig. 9). Besides its advantage of increasing the number of SIFT correspondences due to denser signals in each partial-MIP slice compared to single slices of the original stack, it also reduces the impact of rotation in the directions other than the one studied. The thickness of the partial MIP is determined by the parameter MIP size (in slices) in Fig. 6 . This parameter is taken into account by performing the re-slicing step slice by slice and then computing partial MIP on all the blocks of the specified size parameter from resliced front and back overlapping data portions. The effect of using partial MIP in this specific step will be discussed in the results section.

The set of three registration rounds can be repeated as many times as necessary to further improve the precision of registration. Multiple application of translation and rotation would result in the accumulation of image degradation caused by recalculation and interpolation of voxels at each registration step. To avoid this we combined the translation and rotation parameters calculated by all the previous steps and applied them

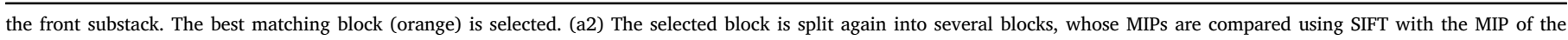

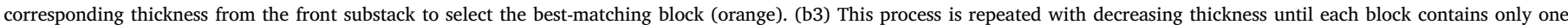

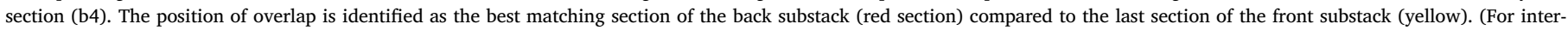
pretation of the references to colour in this figure legend, the reader is referred to the web version of this article.) 
a

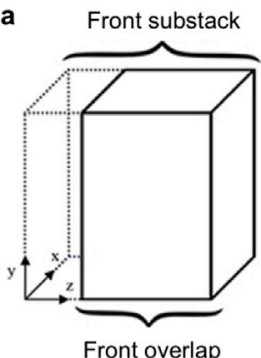

c

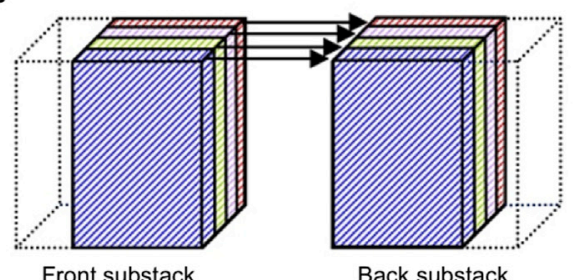

b

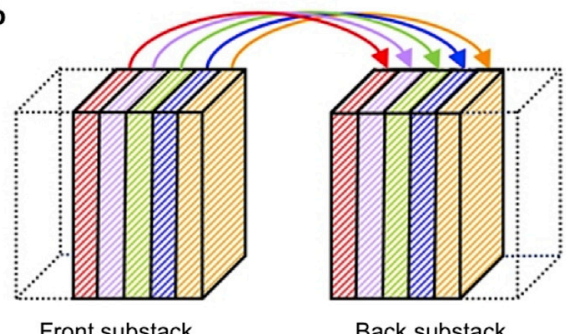

Front substack

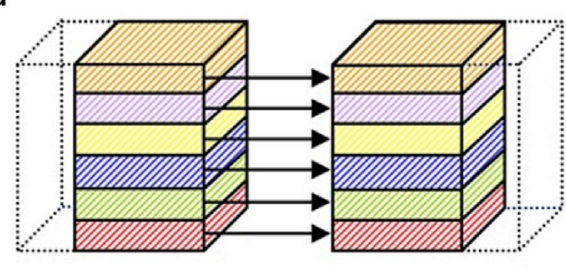

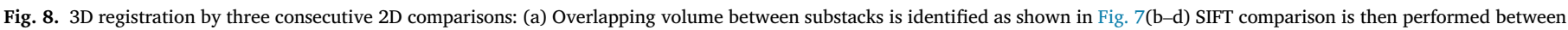
spatially corresponding pairs of partial-MIP slices, first in the $x-y$ plane (b) and then $y-z$ plane (c) and finally $x-z$ plane (d).

a
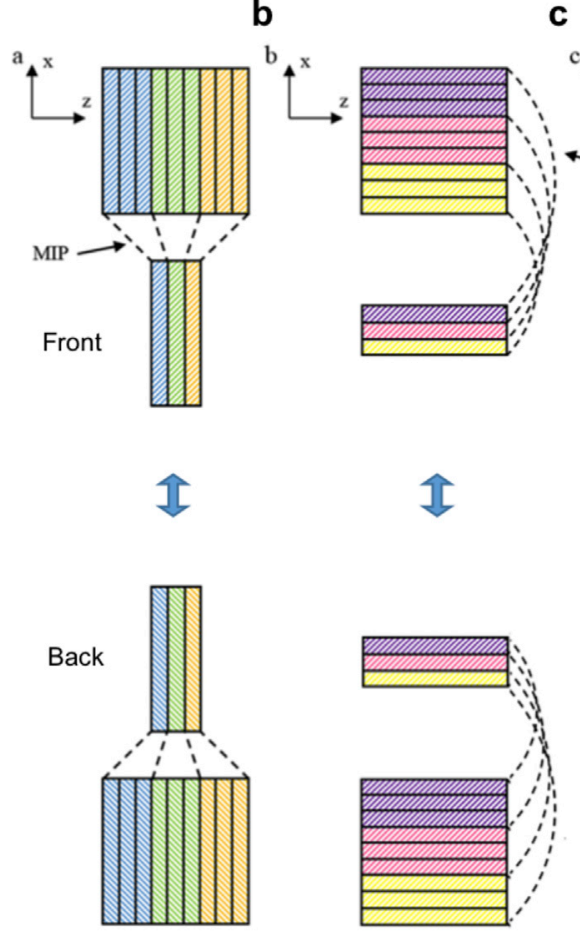

Fig. 9. Creation of partial-MIP slices along $\mathrm{z}, \mathrm{x}$ and $\mathrm{y}$. The image stack volume is re-sliced into three directions, and MIP is calculated from subsets of slices. (a) Partial MIP parallel to x-y plane. (b) Partial MIP parallel to y-z plane. (c) Partial MIP parallel to x-z plane. Each partial-MIP slice contains more signals for feature extraction compared to thin single sections. Reduction of the total number of slices for comparison also reduces computation time.

Registered overlapping portions fusion. Finally a fusion step enables to combine information from the two registered overlapping portions into a single image stack. It allows fixing the difference of brightness within the overlap due to light attenuation by tissue and photobleaching of signals during image acquisition. For this step, we use the stitching plugin developed by Stephan Preibisch (http://imagej.net/Imagestitching). Following this implementation, different fusion methods such as Average, Maximum, Minimum and Linear blending of the two substacks can be selected.

\subsection{In silico simulation of laser-scanning microscopy image stacks}

To quantitatively evaluate our registration algorithm, we need a set of sample image substacks for which degrees of rotation and tilting are precisely known. Such quantitative comparison is difficult on real samples, because image stacks contain uncontrollable factors such as noise, signal attenuation and photobleaching, making them difficult to register precisely with human eyes. An alternative approach is to use in silico simulation to generate images for the evaluation of our registration algorithm. This approach starts by analyzing the noise in laser scanningmicroscope images.

Noise analysis. The noise in 3D image stacks acquired by confocal laser-scanning fluorescence microscopy is known to follow a Gamma law of shape $\mathrm{k}$ and scale $\theta$ [39]:

$f(x ; k ; \theta)=x^{k-1} \frac{\exp (x / \theta)}{\theta^{k} \Gamma(k)}$,

for $x>\theta$ and $k>0$ and $\theta>0$. It must be emphasized that the parameters $\mathrm{k}$ and $\theta$ of the gamma law are not the same in the signal and in the background and are depth-dependent. Thus, we estimated those parameters for two different parts of the image: the signal (in our case neuronal fibers) and the background. For this purpose, we made a binary mask of neuronal fibers by thresholding each section of our real image stacks (Fig. 10a). The threshold was set automatically (Fig. 10b) using Li entropy method [40]. The fiber signal is then extracted by multiplying the corresponding binary mask with the original stacks (Fig. 10c); whereas the background signal is obtained by multiplying the inverse of the binary mask with the original stacks (Fig. 10d).

To evaluate the nature of the noise observed in our datasets, the resulting fiber signal and background images were fitted to a gamma distribution to estimate the parameters $\mathrm{k}$ and $\theta$ throughout the sections of the front and back substacks in five different brain samples (Figs. 11 and 12). Noise in the fiber signal of the front substacks shows a symmetric evolution for $\mathrm{k}$ and $\theta$ (Fig. 11a): while $\mathrm{k}$ is increasing, $\theta$ is decreasing with the depth. In short, the increase of the shape parameter $\mathrm{k}$ with the depth traduces the convergence of the shape of the Gamma distribution towards a Gaussian distribution and the decrease of scale parameter $\theta$ with the depth is coherent with the global decrease of the signal intensity observed in deep layers of the image stacks. Noise in the background shows similar tendency as in the signal part (Fig. 11b). However, the $\mathrm{k}$ parameter is much greater in the background, thus the background noise tends to be more Gaussian. On the contrary, the $\theta$ parameter is more than 

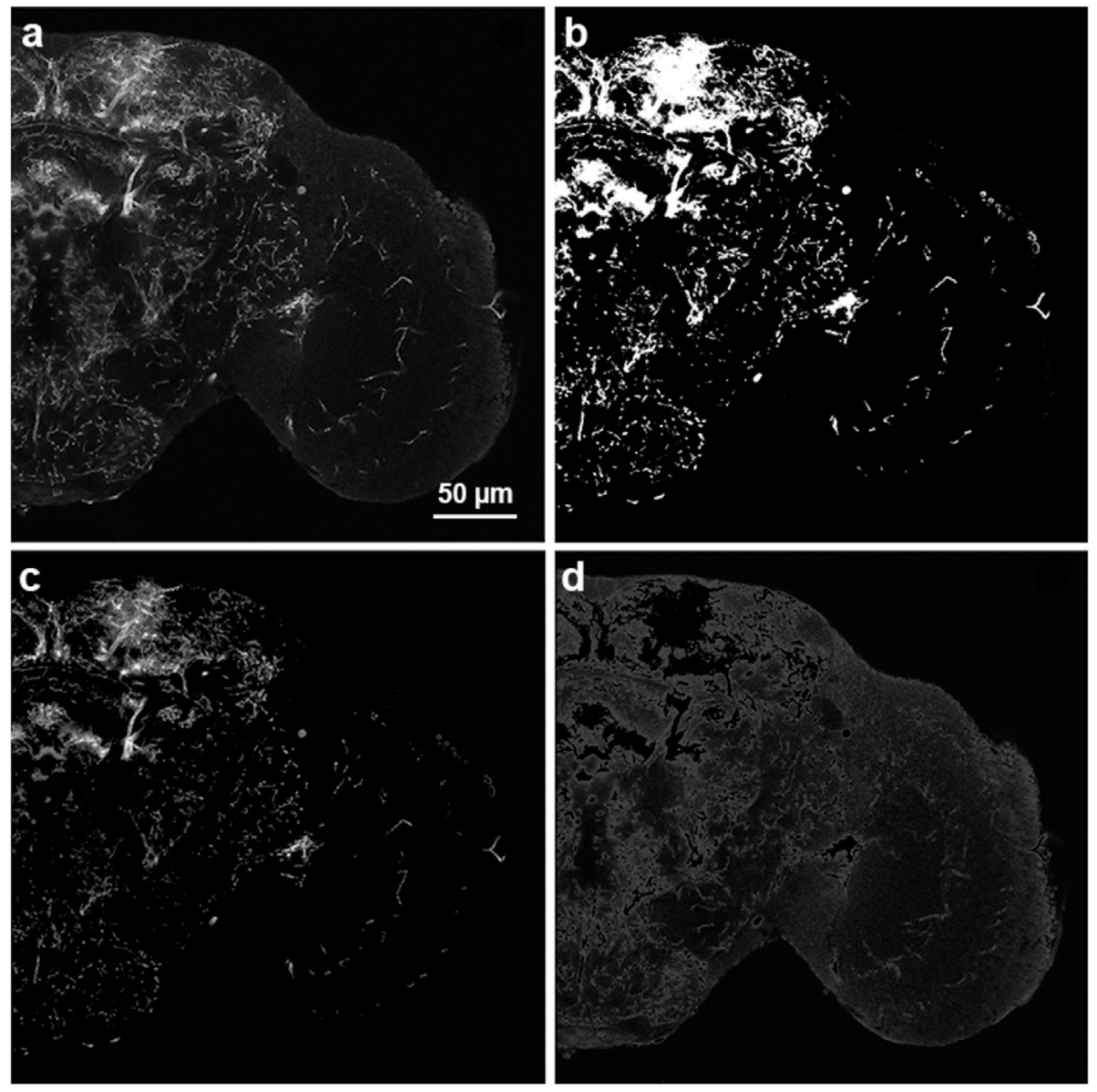



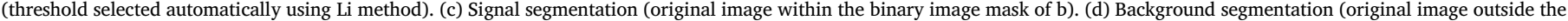
binary image mask of $b$ ).

10 times smaller in the background compared to the signal, which means that the signal distribution is much less spread out.

Noise in the signal and background parts of the back substacks shows similar evolution (Fig. 12): while $\mathrm{k}$ is increasing, $\theta$ is decreasing with the depth in the same order of magnitude. However in the overlap (part of the sample that was already imaged from the frontal surface) $\theta$ is reduced due to photobleaching. Using these data we estimated the noise parameters $\mathrm{k}$ and $\theta$ as the average of the fitting curves of the five different brain samples in the signal and background parts for the front and back substacks (Figs. 11 and 12). These averaged $\mathrm{k}$ and $\theta$ parameters estimated through the depth can then be used as empirical laws for the evolution of noise in laser-scanning microscope modeled as a depth dependent gamma distribution.

Simulation. Based on the previous noise model, we created an in silico simulator of laser-scanning microscopy image stacks (Fig. 13). We first took the binary masks of the signal (i.e. fibers) and background structures (Fig. 13 left) generated for the study of the noise (Fig. 10). We then added two different types of simulated noise on the signal and background structures, respectively, depending on the depth from the surface of the virtual sample (Fig. 13 middle). Finally, signal and background images with added noises were merged to produce simulated laser-scanning microscopy images (Fig. 13 right).

In this study, we simulated a brain sample with a thickness of $120 \mu \mathrm{m}$ (Fig. 14 top, $800 \times 800 \times 300$ voxels). We divided the sample into two overlapping substacks (Fig. 14a top) and added depth-dependent noise to the sample regarding the structure parts and the nature of the substack (i.e., front or back). In these simulated substacks, the red-colored section in (Fig. 14a bottom) is respectively $30 \mu \mathrm{m}$ and $90 \mu \mathrm{m}$ away from the surface of the sample in the front and back substacks. Therefore it appears relatively clear in the front substack (Fig. 14d), but more blurred in the back substack (Fig. 14e). These observations are consistent with real laser-scanning images of neuronal fibers. Finally, by rotating and translating these virtual substacks, we are able to create tilted substacks with known transformation parameters to evaluate the performance and the accuracy of our registration algorithm.

Providing synthetic ground truth for binary structures (such as for the fly brain neurons in Fig. 14) is relatively straightforward. Much more effort would be required for textured samples, and, in practice this is not done (i.e. one rather resort to the sole visual inspection on real images to qualitatively assess the registration performance). We provide an illustration on such a textured sample with images of real fly leg in Supplementary data. However for some biological structures, it is possible to generate ground truth numerically from scratch. This is for instance possible for spheroid i.e. cellular spherical aggregates of cells which constitute 3D in vitro models for life sciences (see Supplementary data for images of real spheroid). Synthetic models of spheroid exist in the literature [41] and to further illustrate the genericity of our simulator we provided additional quantitative analysis with experiments on synthetic spheroids as illustrated in Supplementary material section 4.

\section{Results}

Detailed illustrations are given on the results of the experiments carried on simulated or real fruit fly brains in this section. Supplementary 

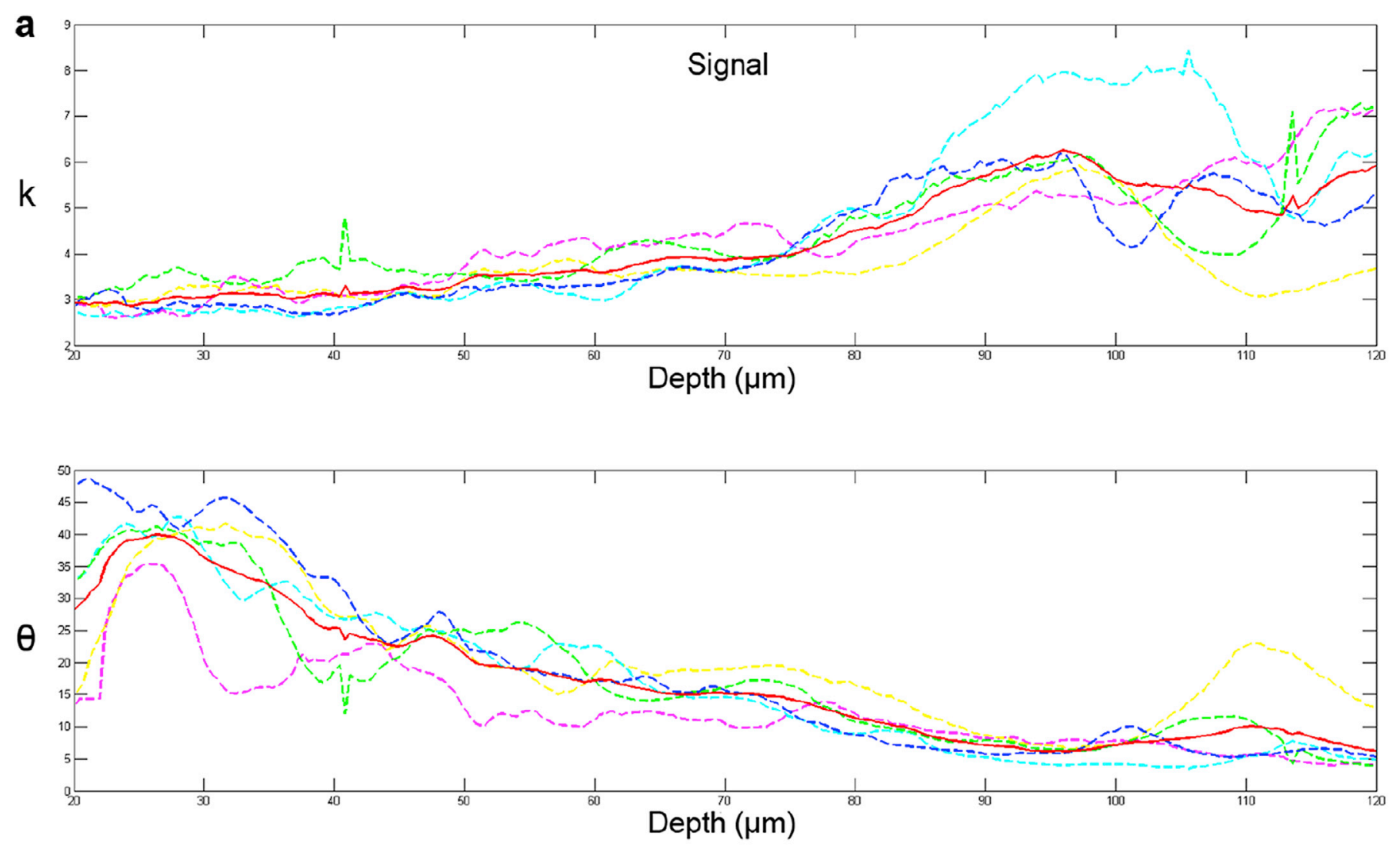

b
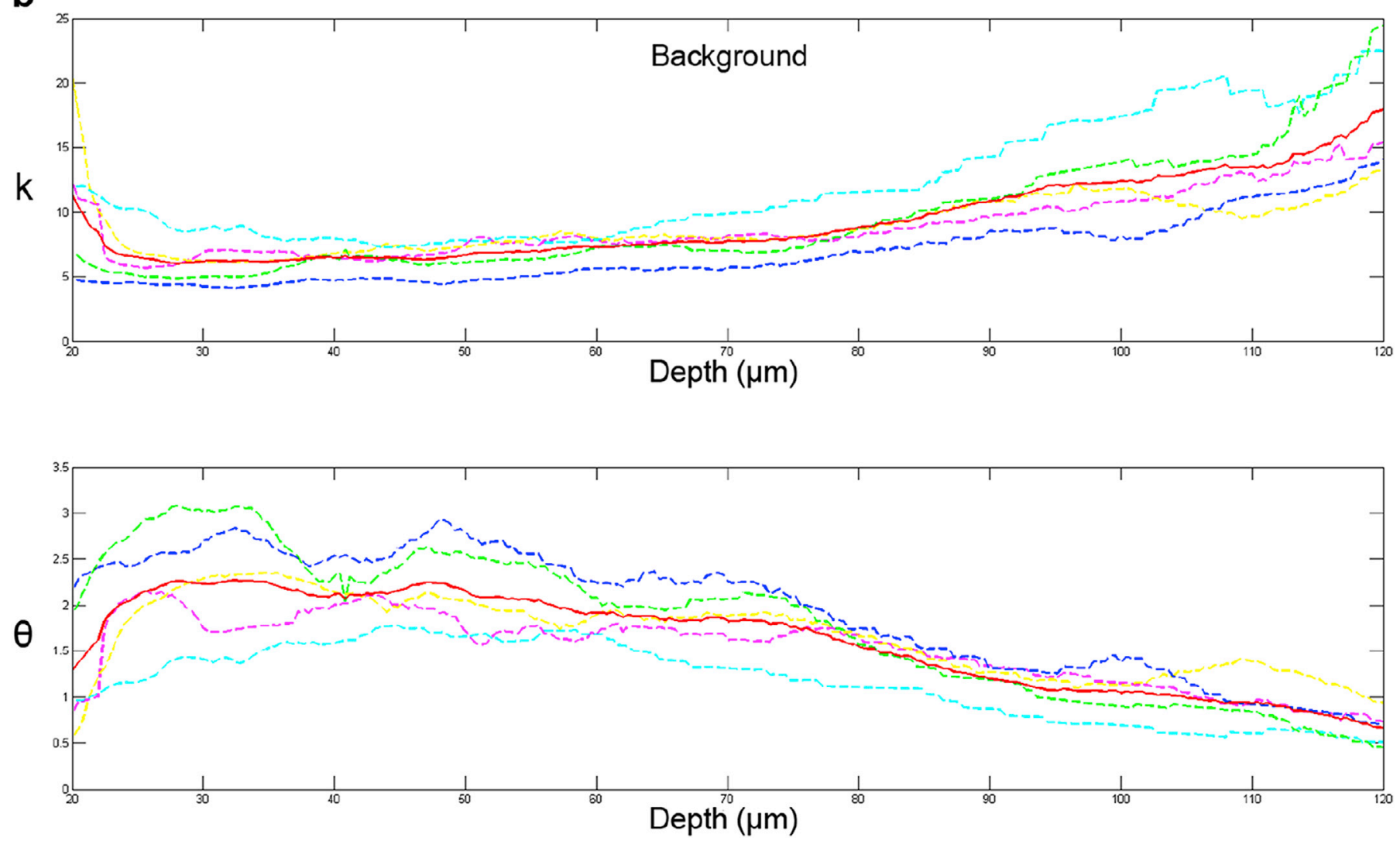

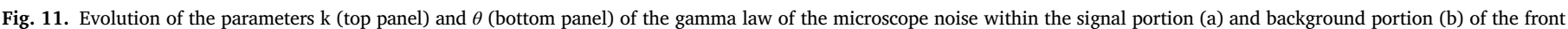

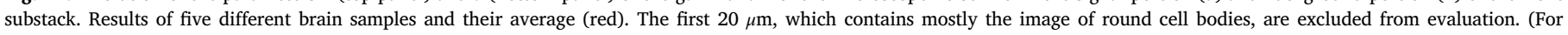
interpretation of the references to colour in this figure legend, the reader is referred to the web version of this article.) 



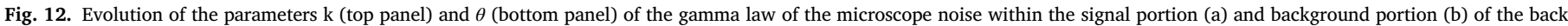


reader is referred to the web version of this article.) 


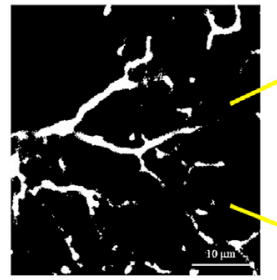

Binary mask of signal structure

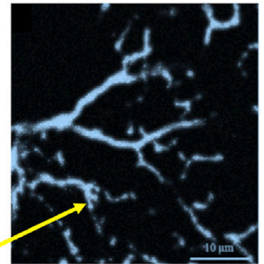

Signal structure

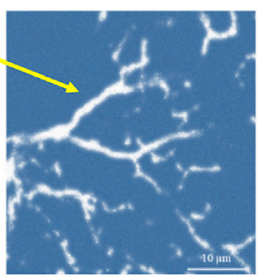

Background structure
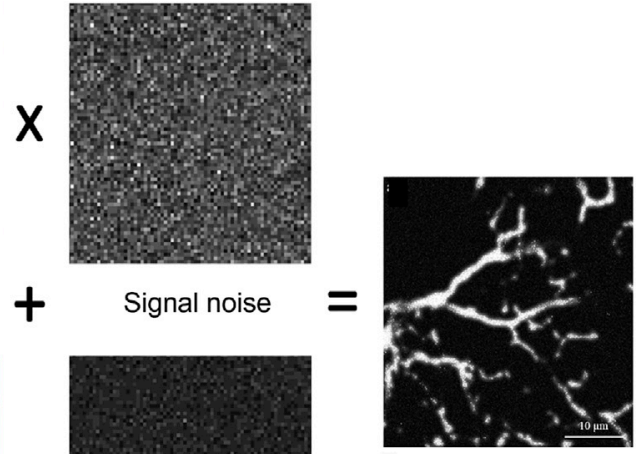

Simulated image

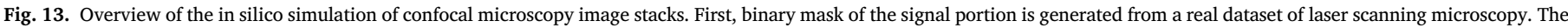

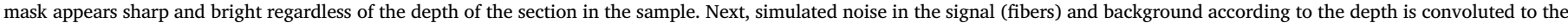
binary masks. Finally, the signal and background images are merged.

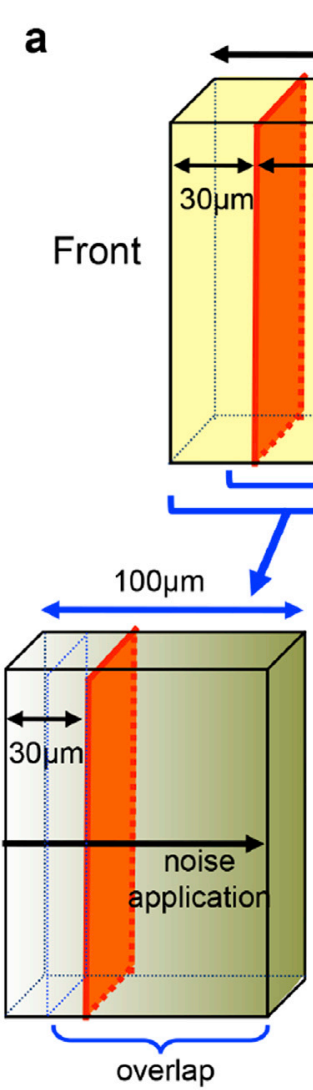

Front substack
$120 \mu \mathrm{m}$
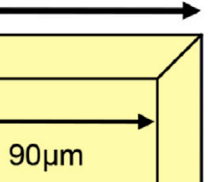

(2)

Back

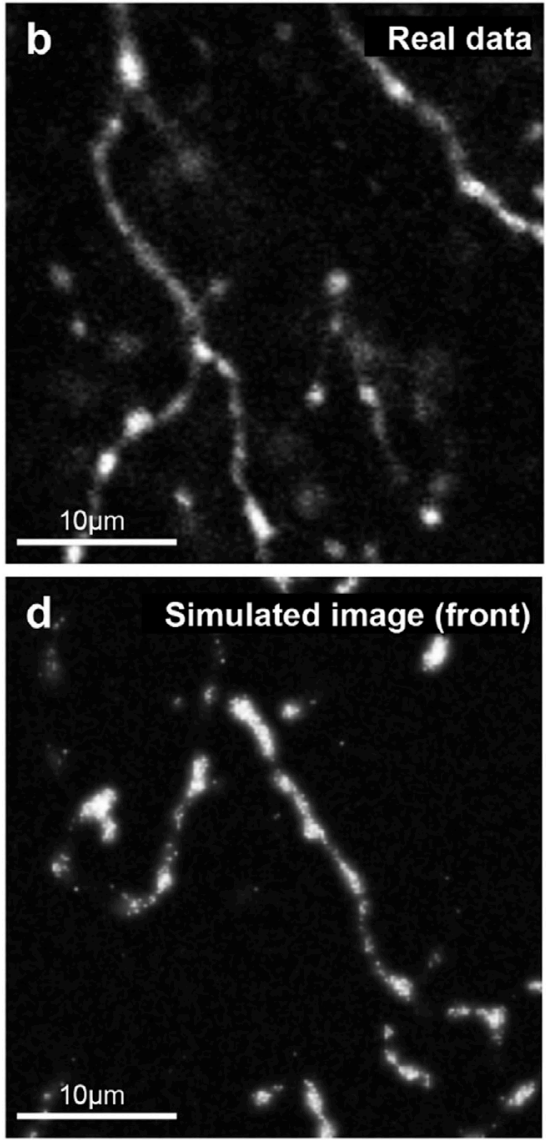
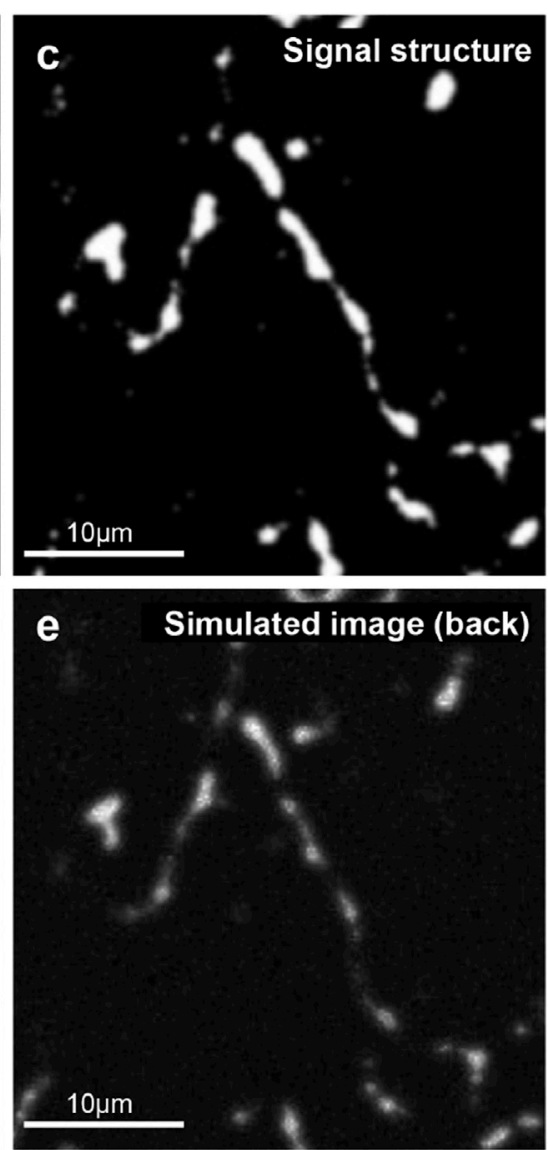

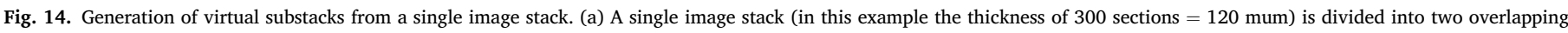

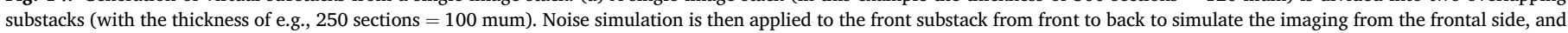

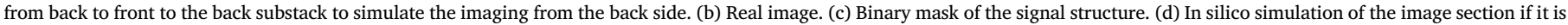

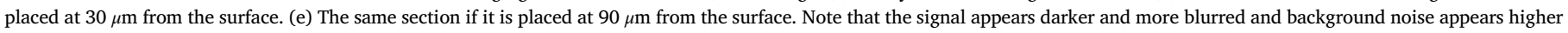
in (e) compared to (d), which is consistent with the image degradation in real confocal microscopy image stacks.

experiments successfully carried on real and simulated spheroids and real fly legs are given in the supplementary data so as to illustrate the generic value of the proposed method.

\subsection{Validation of registration accuracy}

Using the simulator, we tested the quality of our registration algorithm (Figs. 15 and 16). We rotated the virtual back substack by $1^{\circ}$ 

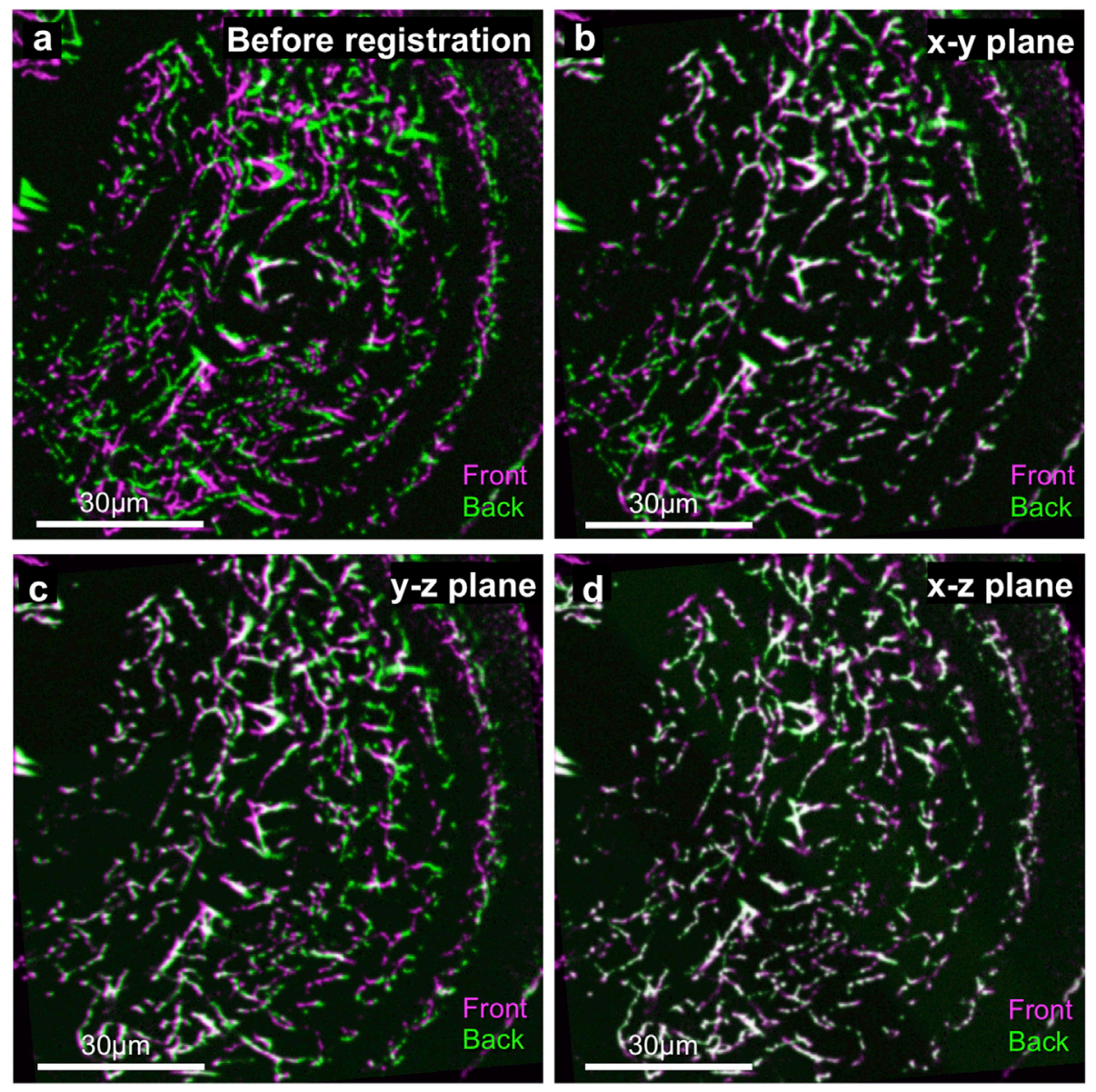

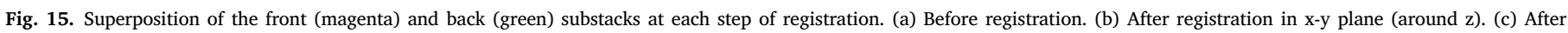

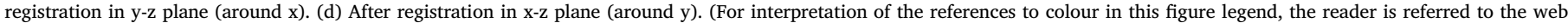
version of this article.)

around $\mathrm{x}-, 2^{\circ}$ around $\mathrm{y}$-, and $5^{\circ}$ around $\mathrm{z}$-axes, applied the noise simulator, and subjected the data to 2D-SIFT-in-3D-Space registration. The performance of our algorithm can be qualitatively assessed in Fig. 15. The front substack in green is superposed to the back substack in magenta. The superposed image should appear white where the two substacks are perfectly registered. The two images do not match just after the overlap detection step (Fig. 15a). Subsequent registration in the $x-y$ plane (Fig. 15b) and then x-z plane (Fig. 15c) and y-z plane (Fig. 15d) gradually improves matching and the overlapping images appear almost completely white with only a slight green or magenta blurred halo, attesting a good quality of registration. The final rotation matrix computed for this dataset is shown in Table 1a. When we convert this rotation matrix into Euler angles $\mathrm{Rx}, \mathrm{Ry}$ and $\mathrm{Rz}$ (respectively rotations around $\mathrm{x}$-, $\mathrm{y}$ - and $\mathrm{z}$-axes), we obtain $\mathrm{Rx}=-1.2757^{\circ}, \mathrm{Ry}=-2.0232^{\circ}$, $\mathrm{Rz}=-5.0010^{\circ}$, which are very similar to the opposite of the angles introduced artificially in our simulated data. Registration was very precise for $\mathrm{z}$ (registration error $0.019 \%)$ and $\mathrm{y}(1.160 \%)$ but worse for $\mathrm{x}(27.566 \%)$.

Registration can be improved by a second loop through the registration process. Indeed, after two iterations of registration, the rotation error around $\mathrm{x}$ is greatly improved (Table $1 \mathrm{~b}$, i.e., $0.428 \%$ ). Higher number of iterations further decreases the rotation errors as well as translation errors, which are lower than $1 \%$ of the original rotation angles and less than 1.3 voxels $(0.26 \mu \mathrm{m})$ of translation. In practice, 2 iterations seem to be a good compromise between accuracy and computation time, because further registrations do not greatly improve the errors. It can be noted however from Table $1 \mathrm{~b}$ that rotation error after multiple iterations becomes very small around z-axis, but remain a bit larger around $\mathrm{x}$ - and $\mathrm{y}$-axes. The better registration accuracy around $\mathrm{z}$ axis compared to $\mathrm{x}$ and $\mathrm{y}$ can be explained by the size of the image slices. Indeed, the $x$-y slices are much larger $(800 \times 800$ pixels $)$ than the $x-z$ and $y-z$ slices $(800 \times 200$ pixels), leading to a greater number of matching features. Registration performance can also be examined using the overlap correspondence curve, which shows the number of matching features between the last section of the front substack and each section of the back substack (Fig. 16a). After the overlap detection (zero iteration), the corresponding curve appears very broad with a small number of matching features (blue line in Fig. 16a). Indeed, the front and back substacks are not registered yet and thus the similarity between them is still rather low. On the contrary, the correspondence curve after the first round of registration is much thinner and sharper, and the number of features is three times larger, traducing a higher similarity between the front and back substacks (see Fig. 16b) and thus the efficiency of the registration. The correspondence curves after the second iteration onwards are essentially superposition of the image stacks, without showing further improvement (Fig. 16c-d). Finally, because image size is likely to affect registration quality, and because image sizes in $\mathrm{x}-\mathrm{z}$ and $\mathrm{y}-\mathrm{z}$ slices are determined by the overlap size, we also investigated its effect. When we reduced the overlap by taking fewer sections from the front and back substacks, registration error increased (Table 1c). If the overlap size was too small (e.g., 50 voxels), the algorithm struggled to find rotation around $\mathrm{x}$ and $\mathrm{y}$ with the default parameters. However, modification of those parameters (here reducing the minimum size of structures to 1) fixed the problem (Table 1c, bottom). These results show that multiple iterations and large enough overlap size are both important for precise registration. 
a - Before registration Iteration $1 \longrightarrow$ Iteration $2 \longrightarrow$ Iteration $3 \longrightarrow$ Iteration 4 Iteration 5
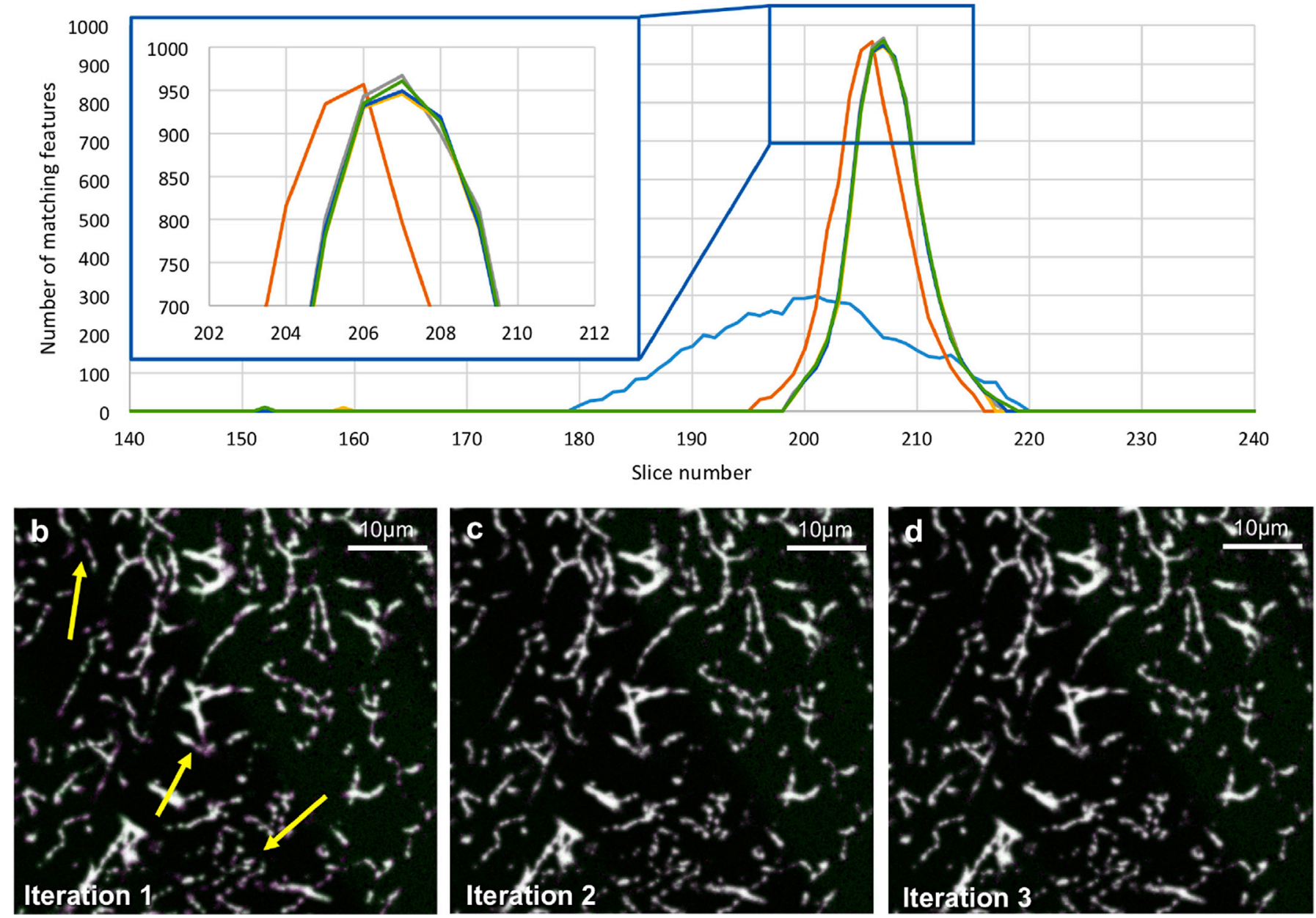

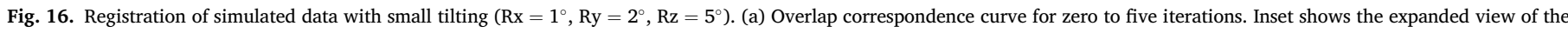

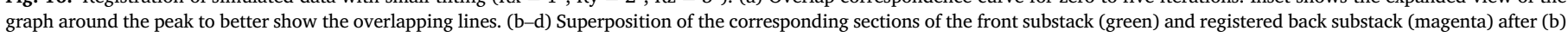
one, (c) two and (d) three iterations. (For interpretation of the references to colour in this figure legend, the reader is referred to the web version of this article.)

\subsection{Performance comparison between block-by-block and slice-by-slice overlap detection}

We evaluated the accuracy of the block-by-block and slice-by-slice overlap detection approaches. Block-by-block approach is meant to allow registration of substacks with large tilting angles. To determine the tilting limit of this approach, we fixed the tilting angles of $\mathrm{Rz}=5^{\circ}$, $\mathrm{Ry}=2^{\circ}$ and gradually increased the value of $\mathrm{Rx}$ by $5^{\circ}$ steps and submitted the data to slice-by-slice (Table 2a) and block-by-block (Table 2b) approaches. The splitting parameter was set at 5 for block-by-block approach. Please note that both approaches used partial MIP in the registration step and that its size was set to 20 slices for both versions. Both overlap detection approaches performed similarly for small tilting angles $\left(<15^{\circ}\right)$ leading to good results. However, stitching with block-byblock approach produced smaller error rates and was two times faster than slice-by-slice approach. The difficulty of overlap detection increases with tilting because the similarity of sections perpendicular to $\mathrm{z}$-axis decreases. Overlap detection with slice-by-slice approach becomes difficult as $\mathrm{Rx}$ reaches $20^{\circ}$, where the correspondence curve does not present the typical bell-shape but is extremely noisy with very few matching features (Fig. 17a). Thanks to the registration step around the three axes, overlap detection was drastically improved after a few registration iterations in terms of matching features (orange curve in Fig. 17b). However, the computed rotation parameters were not as accurate as expected, with high rotation error rate around $\mathrm{x}$ - and $\mathrm{y}$-axes
(6.35\% and $19.1 \%$ respectively, Table 2 a bottom).

Stitching with block-by-block approach at $\mathrm{Rx}=20^{\circ}$ also caused erroneous computed rotation angle, with an error rate as high as $741 \%$ and $79.9 \%$ around $\mathrm{x}$ - and $\mathrm{y}$-axes (Table $2 \mathrm{~b}$, 4th row). Because the thickness of the detected initial overlap was small (77-section thick), the matching features were concentrated only in a small portion of the data (Fig. 18a, green circle). To address this we then decreased the splitting parameter to two, but the detected overlap thickness was again as low as 76 sections (Fig. 18b, green circle), causing similarly high error rates (Table 2b, 5th row). In our algorithm, we first detect the overlapping portion of the substacks and then perform subsequent registration only using this portion of the data. With high tilting angle between substacks, only a small part of the overlapping volume might be detected. However, we can effectively skip overlap detection by setting the splitting parameter to one (i.e., no splitting) so that the program uses the data of the entire substacks to compute registration (Fig. 18c, green circle). This strategy resulted in much improved rotation error as low as $0.32 \%$ (Table 2b, last row).

\subsection{Influence of the splitting parameter in block-by-block approach}

We next analyzed the effect of the splitting parameter for block-byblock overlap detection for a moderate tilting angle of $\mathrm{Rz}=5^{\circ}$, $\mathrm{Ry}=2^{\circ}$ and $\mathrm{Rx}=5^{\circ}$ (Table 3 ). We performed registration for splitting parameter ranging from 1 (no split) to the number of the sections in the 
Table 1

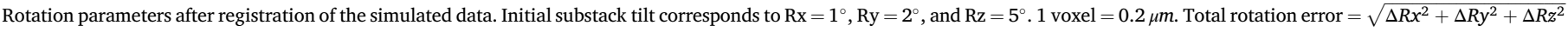
and Total translation error $=\sqrt{\Delta x^{2}+\Delta y^{2}+\Delta z^{2}}$.

\begin{tabular}{|c|c|c|c|c|c|c|c|c|c|}
\hline \multicolumn{10}{|c|}{ a: Conversion matrix (Substack size $800 \times 800 \times 250$, i.e., overlap: 200 , after single iteration) } \\
\hline \multirow{4}{*}{\multicolumn{5}{|c|}{$\begin{array}{l}0.9955825655501388 \\
-0.08711895143914962 \\
0.03532588980227439 \\
R x=-1.2757 \mathrm{Ry}=-2.0232 \mathrm{Rz}=-5.0010\end{array}$}} & \multicolumn{3}{|c|}{0.08765056866153828} & & \multirow{4}{*}{$\begin{array}{l}-0.03262316976298832 \\
0.023091300973873324 \\
0.9997414741550107\end{array}$} \\
\hline & & & & & 0.99578 & 605004061 & & & \\
\hline & & & & & -0.0222 & 38860907968 & & & \\
\hline & & & & & & & & & \\
\hline \multicolumn{10}{|c|}{ b: Registration results after different number of iterations (Substack/overlap size are the same as above) } \\
\hline \multirow[t]{2}{*}{ Iter \# } & \multicolumn{4}{|c|}{ Euler angles: } & \multicolumn{3}{|l|}{ Rotation error: } & \multirow[t]{2}{*}{ Total rot. error (\%) } & \multirow[t]{2}{*}{ Total trans. error (voxels) } \\
\hline & \multicolumn{2}{|l|}{$\mathrm{Rx}\left({ }^{\circ}\right)$} & Ry $\left({ }^{\circ}\right)$ & $\mathrm{Rz}\left({ }^{\circ}\right)$ & $\Delta \mathrm{Rx}(\%)$ & $\Delta$ Ry (\%) & $\Delta \mathrm{Rz}(\%)$ & & \\
\hline 1 & -1.2757 & & & -5.0010 & 27.566 & 1.160 & 0.019 & 27.590 & 1.898 \\
\hline 2 & -0.9957 & & & -4.9998 & 0.428 & 0.734 & 0.004 & 0.850 & 1.292 \\
\hline 3 & -0.9914 & & & -5.0043 & 0.855 & 0.461 & 0.085 & 0.975 & 1.243 \\
\hline 4 & -0.9902 & & & -5.0015 & 0.976 & 0.674 & 0.030 & 1.186 & 1.221 \\
\hline 5 & -0.9927 & & & -5.0004 & 0.733 & 0.560 & -0.009 & 0.922 & 0.928 \\
\hline \multicolumn{10}{|c|}{ c: Registration results for different amount of overlap (Results after 2 iterations) } \\
\hline \multirow{2}{*}{\multicolumn{2}{|c|}{ Overlap (voxels) }} & \multicolumn{3}{|c|}{ Euler angles: } & \multicolumn{3}{|c|}{ Rotation error: } & \multirow[t]{2}{*}{ Total rot. error (\%) } & \multirow[t]{2}{*}{ Total trans. error (voxels) } \\
\hline & & $\mathrm{Rx}\left({ }^{\circ}\right)$ & Ry $\left({ }^{\circ}\right)$ & $\mathrm{Rz}\left({ }^{\circ}\right)$ & $\Delta \mathrm{Rx}(\%)$ & $\Delta$ Ry $(\%)$ & $\Delta \mathrm{Rz}(\%)$ & & \\
\hline 200 & & -0.9957 & -1.9853 & -4.9998 & 0.428 & 0.734 & 0.004 & 0.850 & 1.292 \\
\hline 150 & & -1.0029 & -1.9854 & -4.9982 & 0.292 & 0.730 & 0.036 & 0.787 & 1.867 \\
\hline 100 & & -1.0259 & -1.9925 & -5.0006 & 2.594 & 0.377 & 0.012 & 2.621 & 2.895 \\
\hline 50 & & -1.0122 & -2.0001 & -5.0106 & 1.218 & 0.007 & 0.213 & 1.237 & 3.884 \\
\hline
\end{tabular}

Table 2

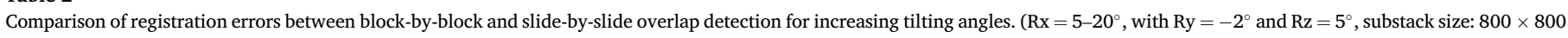
x 250. Partial MIP size is set at 20 slices. Computation time was measured on a computer with an Intel Core i7-4930 k processor (6-core) running at 3.4 GHz).

\begin{tabular}{|c|c|c|c|c|c|c|c|c|c|c|c|}
\hline \multicolumn{12}{|c|}{ a: Slice-by slice overlap detection } \\
\hline \multirow[t]{2}{*}{ Tilt $\left({ }^{\circ}\right)$} & \multicolumn{4}{|c|}{ Euler angles: } & & \multicolumn{3}{|l|}{ Rotation error: } & \multirow[t]{2}{*}{ Total rot. error (\%) } & \multirow[t]{2}{*}{ Total trans. error (voxels) } & \multirow[t]{2}{*}{ Comput. Time (min) } \\
\hline & $\mathrm{Rx}\left({ }^{\circ}\right)$ & \multicolumn{2}{|c|}{$\operatorname{Ry}\left({ }^{\circ}\right)$} & \multicolumn{2}{|c|}{$\mathrm{Rz}\left({ }^{\circ}\right)$} & $\Delta \mathrm{Rx}(\%)$ & $\Delta$ Ry (\%) & $\Delta \mathrm{Rz}(\%)$ & & & \\
\hline 5 & -4.99 & 2.00 & & -4.99 & & 0.036 & 0.180 & 0.007 & 0.18 & 2.38 & 71 \\
\hline 10 & -9.99 & 2.01 & & -5.00 & & 0.084 & 0.566 & 0.017 & 0.57 & 4.50 & 53 \\
\hline 15 & -14.98 & 2.00 & & -5.00 & & 0.081 & 0.086 & 0.046 & 0.12 & 6.54 & 45 \\
\hline 20 & -18.72 & -1.6 & & -4.96 & & 6.352 & 19.05 & 0.616 & 20.09 & 12.13 & 37 \\
\hline \multicolumn{12}{|c|}{ b: Block-by-block overlap detection } \\
\hline \multirow[t]{2}{*}{ Tilt $\left({ }^{\circ}\right)$} & \multirow[t]{2}{*}{ Split } & \multicolumn{4}{|c|}{ Euler angles: } & \multicolumn{3}{|c|}{ Rotation error: } & \multirow[t]{2}{*}{ Total rot. error (\%) } & \multirow[t]{2}{*}{ Total trans. error (voxels) } & \multirow[t]{2}{*}{ Comput. Time (min) } \\
\hline & & $\mathrm{Rx}\left({ }^{\circ}\right)$ & $\operatorname{Ry}\left({ }^{\circ}\right)$ & & $\mathrm{Rz}\left({ }^{\circ}\right)$ & $\Delta \mathrm{Rx}(\%)$ & $\Delta$ Ry (\%) & $\Delta \mathrm{Rz}(\%)$ & & & \\
\hline 5 & 5 & -4.99 & 2.00 & & -4.99 & 0.012 & 0.126 & 0.049 & 0.13 & 2.38 & 40 \\
\hline 10 & 5 & -9.99 & 2.00 & & -4.99 & 0.090 & 0.356 & 0.095 & 0.48 & 4.46 & 26 \\
\hline 15 & 5 & -14.97 & 2.00 & & -5.00 & 0.162 & 0.125 & 0.079 & 0.22 & 6.65 & 23 \\
\hline 20 & 5 & -168.2 & -1.24 & & -5.44 & 741.2 & 37.8 & 8.8 & 742.3 & 16124 & 10 \\
\hline 20 & 2 & 88.93 & 58.6 & & -88.4 & 544.6 & 2832.8 & 1669.8 & 3333.1 & 368420 & 11 \\
\hline 20 & 1 & -19.93 & 2.00 & & -5.01 & 0.319 & 0.126 & 0.242 & 0.42 & 8.78 & 33 \\
\hline
\end{tabular}

substack (corresponding to the slice-by-slice approach). After 5 iterations, registration accuracy was essentially the same for all splitting parameters (Table 3), but it should be noted that computation time was long when the split value $=1$ (58 $\mathrm{min}$ ) because the entire substack data are subjected to subsequent registration. Because the overlap does not cover all slices in case of moderate tilting angles, many SIFT features will not match and their consideration does not bring any improvement to the registration result. Because of this, a split value of 1 must be used only in case of high tilting between the two stacks as discussed in the previous subsection.

When splitting was activated (split value ${ }_{i} 1$ ), computation time depended on the total number of SIFT comparisons for overlap detection (Table 3, second column). This comparison number is linked to the split value. For our simulated substacks ( 250 sections thick) and a split value of 5 , the program first splits the substacks into 5 blocks of 50 sections and does 5 SIFT comparisons (between the last block of the front substack and the 5 blocks of the back substack) to find the best match. The selected block is then split into 5 blocks of 10 sections and compared with a block of the same size from the front substack ( 5 comparisons). The best- matching block is further split into 5 blocks of 2 sections and once again compared five times. Because the new selected block contains only 2 sections, it cannot be further split and a slice-by-slice comparison is used to determine the exact position of overlap (two comparisons). Thus, we need in total 17 comparisons to detect the overlap size. This number of comparisons is much smaller than that of the slice-by-slice comparison (250). However, the computation time was only about two times faster (40 min versus $71 \mathrm{~min}$, Table 3). This is likely because thick partial MIP images present more SIFT candidates. The number of comparison increases as we set higher splitting parameter, which results in longer computation time (Table 3). Thus, for practical purposes splitting parameter between 2 and 5 should be preferred. However, if the sections contain very dense signals, setting a too small split value may cause saturation or too many unmatching features. The running time then increases a lot but not the quality of registration.

\subsection{Impact of image filtering on registration accuracy}

As previously discussed in introduction, signal quality tends to 

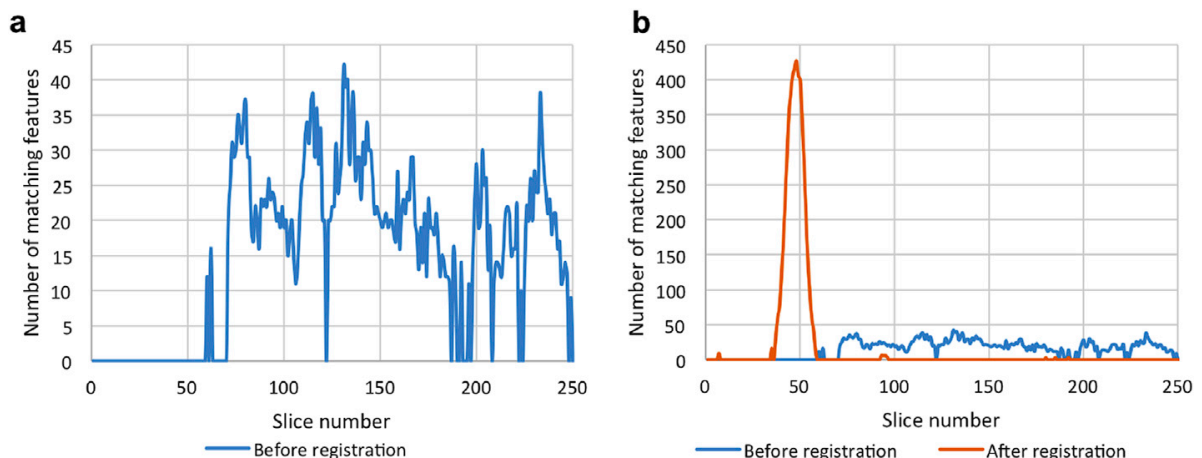

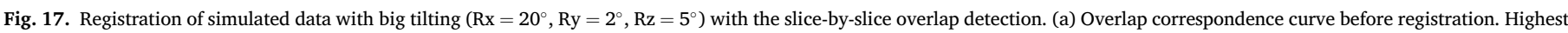
peak was at the 131st section. (b) Comparison of the curves before and after registration. Actual matching peak after registration was at the 49th section.

a $\quad$ Splitting $=5$

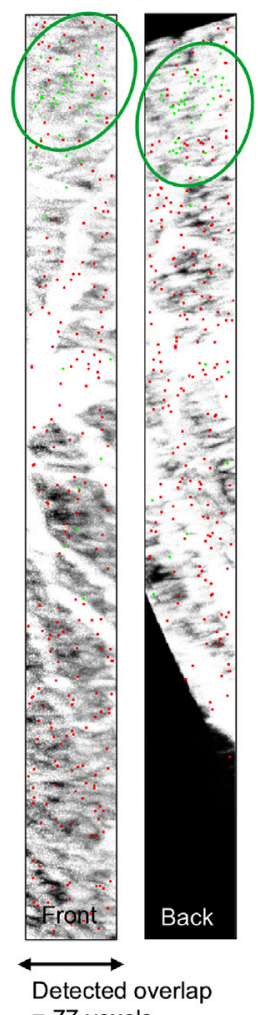

b $\quad$ Splitting $=2$

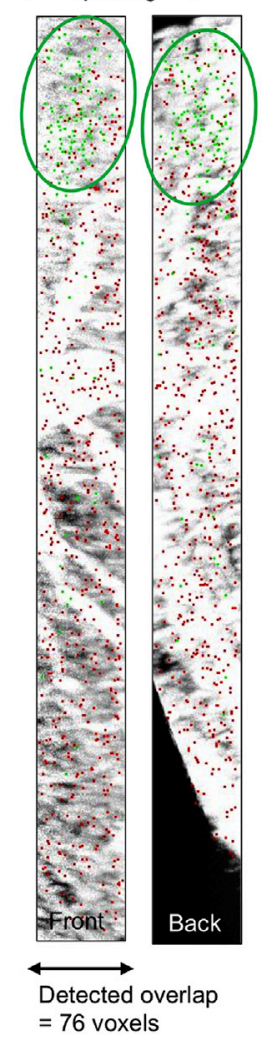

C $\quad$ Splitting $=1$ (no split)

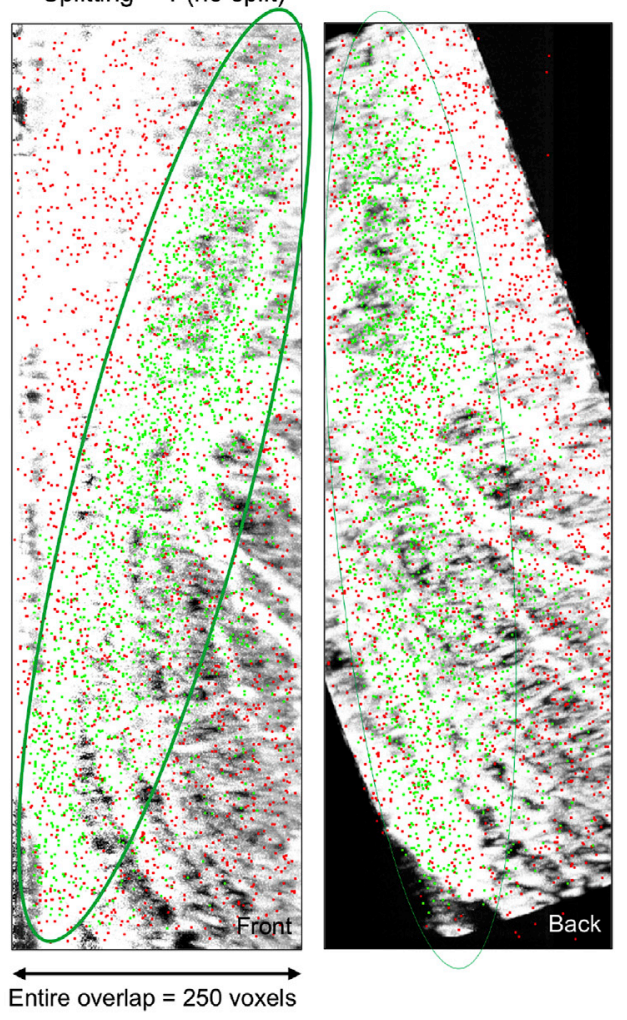

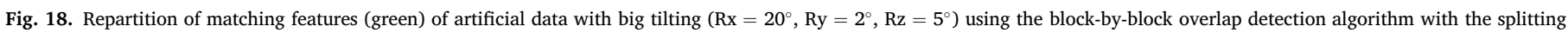

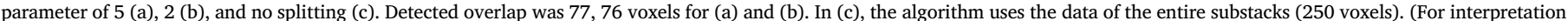
of the references to colour in this figure legend, the reader is referred to the web version of this article.)

Table 3

Registration errors and computation time of the block-by-block algorithm for different splitting parameters with $\mathrm{Rx}=5^{\circ}, \mathrm{Ry}=-2^{\circ}$ and $\mathrm{Rz}=5^{\circ}$. Partial MIP size is set at 20 slices.

\begin{tabular}{|c|c|c|c|c|c|c|c|c|c|c|}
\hline \multirow[t]{2}{*}{ Split } & \multirow[t]{2}{*}{ Compa-rison \# } & \multicolumn{3}{|c|}{ Euler angles: } & \multicolumn{3}{|c|}{ Rotation error: } & \multirow[t]{2}{*}{ Total rot. error (\%) } & \multirow[t]{2}{*}{ Total trans. error (voxels) } & \multirow[t]{2}{*}{ Comput. Time (min) } \\
\hline & & $\mathrm{Rx}\left({ }^{\circ}\right)$ & Ry $\left({ }^{\circ}\right)$ & $\mathrm{Rz}\left({ }^{\circ}\right)$ & $\Delta \mathrm{Rx}(\%)$ & $\Delta$ Ry $(\%)$ & $\Delta \mathrm{Rz}(\%)$ & & & \\
\hline $1^{\mathrm{a}}$ & 1 & -4.99 & 2.003 & -4.9977 & 0.061 & 0.162 & 0.046 & 0.180 & 2.38 & 58 \\
\hline 2 & 15 & -4.997 & 2.000 & -5.001 & 0.061 & 0.031 & 0.034 & 0.076 & 2.44 & 32 \\
\hline 5 & 17 & -4.999 & 2.002 & -4.997 & 0.012 & 0.126 & 0.049 & 0.136 & 2.38 & 40 \\
\hline 10 & 22 & -4.998 & 2.000 & -4.998 & 0.028 & 0.023 & 0.024 & 0.042 & 2.40 & 44 \\
\hline 15 & 31 & -4.991 & 1.999 & -5.001 & 0.164 & 0.028 & 0.030 & 0.169 & 2.38 & 46 \\
\hline 20 & 32 & -4.999 & 2.005 & -5.001 & 0.013 & 0.271 & 0.033 & 0.273 & 2.49 & 48 \\
\hline 50 & 55 & -4.998 & 2.003 & -4.998 & 0.032 & 0.160 & 0.035 & 0.167 & 2.37 & 60 \\
\hline 125 & 76 & -4.998 & 2.004 & -4.998 & 0.040 & 0.199 & 0.029 & 0.205 & 2.42 & 125 \\
\hline $250^{\mathrm{b}}$ & 71 & -4.998 & 2.003 & -4.999 & 0.036 & 0.180 & 0.007 & 0.184 & 2.38 & 250 \\
\hline
\end{tabular}

\footnotetext{
${ }^{\text {a }}$ No split.
}

${ }^{\mathrm{b}}$ Effectively the same as slice-by-slice comparison. 

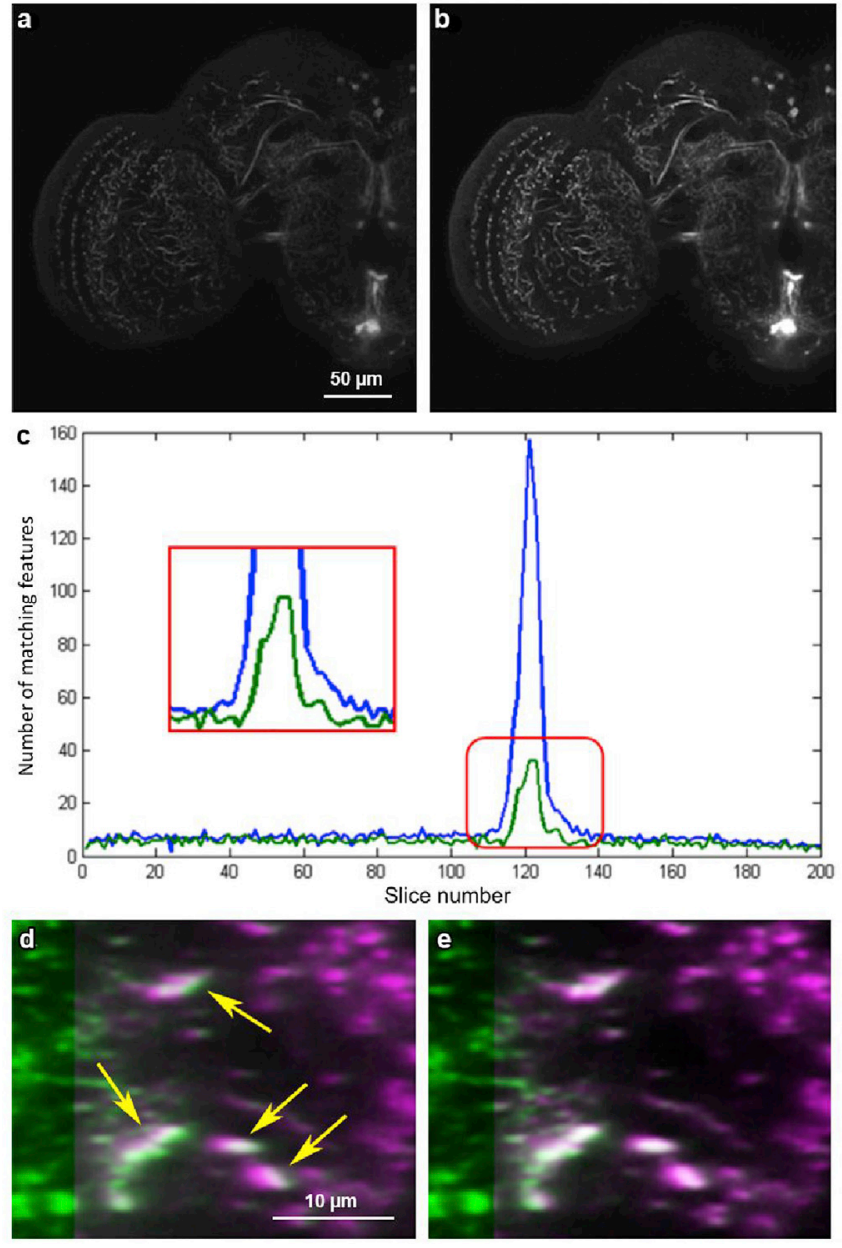

Fig. 19. Improved registration after intensity and gamma correction. (a) Original data. (b) Corrected data (Intensity 100\%-300\% linear, gamma 1 to 1.4 linear). (c) Number of SIFT features in the overlap detection for original data (green) and intensity/gamma corrected data (blue). Rectangle shows the plateau of the matching peak without intensity and gamma correction. (d, e) The $\mathrm{x}-\mathrm{z}$ cross-sections of the stack, showing superposition of the front (green, left side of the panels) and back substack images (magenta, right side of the panels). Registration on original data (d) and corrected data (e). Brightness is enhanced for better visualization. Arrows in (d) indicate green and magenta fringe caused by the mismatch between the front and back substacks, which are hardly seen in (e). Scale bars $=50 \mu \mathrm{m}$ (a, b), $10 \mu \mathrm{m}$ (d, e). (For interpretation of the references to colour in this figure legend, the reader is referred to the web version of this article.)

decrease with the depth in the samples. Unless adequately compensated, images of the sections that are deep in the specimen tend to appear darker than those that are close to the sample surface because of the attenuation of light passing through the specimen and photobleaching of fluorophores during image acquisition [5]. Despite the relative robustness of SIFT matches toward brightness difference between the two images to be registered, it can be helpful to perform a filtering step of the image substacks to compensate the brightness mismatch of comparable sections before registration. To address this problem we developed a Fiji/ImageJ plugin Progressive Intensity and Gamma Correction (see Figs. S4 and S5 of the Supplementary data). Using this plugin we tested the effect of intensity/gamma correction on the precision of registration. Fig. 19 compares the results of overlap extraction for the same pair of substacks with or without intensity/gamma correction. Compared to the original substack (Fig. 19a), intensity was corrected between $100 \%$ and $300 \%$ linearly and gamma between 1.0 and 1.4 linearly from the first (shallowest) to the last (deepest) sections of substacks (Fig. 19b). After the overlap detection (before registration), the overlap correspondence curve appears much thinner and sharper, and the number of features is five times larger (Fig. 19c) after intensity/gamma correction (blue line) than with the original data (green line). Indeed, corresponding curve of the original data presents a plateau, making the overlap size determination confusing, whereas the curve of the corrected data shows a sharp peak from which we can precisely deduce the overlap size. This difference can be noticed as a slight shift along the z-axis when the front and back substacks are merged. In the cross section view of the stack (Fig. 19d and e), we can see that the superposition works better (white color without green or magenta fringes) for the intensity-corrected data (Fig. 19e) than for the original data (arrows in Fig. 19d).

\subsection{Performance on real data}

Our plugin can efficiently perform very fine adjustments and precisely register small objects such as neuronal fibers and presynaptic sites. Fig. 20 shows the performance of our registration algorithm for the real image stacks of the Drosophila brain at a voxel resolution of $0.2 \times 0.2 \times 0.2 \mu \mathrm{m}(1600 \times 1600$ voxels and 800 sections $)$. The front substack (Fig. 20a, shown in magenta in Fig. 20c) was registered and superposed to the back substack (Fig. 20b, shown in green in Fig. 20c). In the overlaid image (Fig. 20c), green and magenta cast should appear on the opposite sides of the objects if two stacks are out of alignment. The lack of such cast attests perfect registration. (Note: green or magenta cast that appears on the entire object is caused by the voxel intensity differences between front and back substacks.) The stitched dataset serves as a starting point for characterizing the fine architecture of the visualized neurons at very high resolution.

\subsection{Effect of optical aberrations}

2D-SIFT-in-3D-Space Volume Stitching performs only affine transformation. This is because the section images of the same sample obtained from different sides should have same morphology. However, we found that this assumption may not always be true. In a few cases, we noticed that peripheral areas of the image could not be registered completely even when complete match was achieved in the central parts of the image. There is apparently a slight non-linear distortion between the section images of the same part of the sample obtained from the different substacks (Fig. 21). This is most likely because of the optical aberrations caused by the microscope objectives and scan optics. Among Seidels five aberrations, spherical aberration, coma, and astigmatism mainly affect the sharpness of the image without affecting the location of the signal. Those aberrations may cause uneven sharpness in the image field. Then sharper parts of the image with the highest feature correspondences concentration will have a stronger influence on the model computation, leading to perfect registration in those parts but a shift between the front and back substacks in other parts of the image. Moreover, the other two aberrations field curvature and distortion cause distorted projection from the signal distribution in the sample to the obtained 3D image space, making straight lines in the sample appear curved along z-axis (field curvature) or in the $\mathrm{x}$-y plane (distortion). An objective lens tuned for the best performance at certain focal depth in the sample may not perform optimally at shallower or deeper levels. Thus, the optical sections of the same part of the specimen but obtained from different sides (i.e., at different depth) may not be identical. Adaptive optics and post-imaging distortion correction should be helpful to reduce the effect of optical aberrations [42-44].

\section{Conclusion}

Although confocal laser-scanning fluorescent microscopy is used extensively in many biological laboratories to visualize a large variety of cells and specimens, its depth limitation problem makes it difficult to study thick samples. Even though microscope manufacturers try to provide objective lenses with longer working distances, diffraction and scattering of lights within the optically uneven specimen still causes practical limitations. Two-photon microscopy does not solve this 



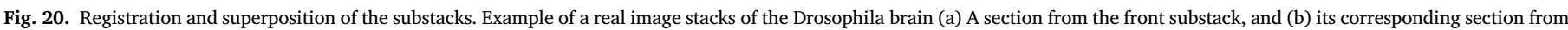

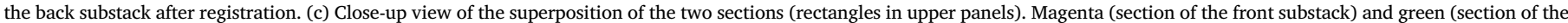


this article.)
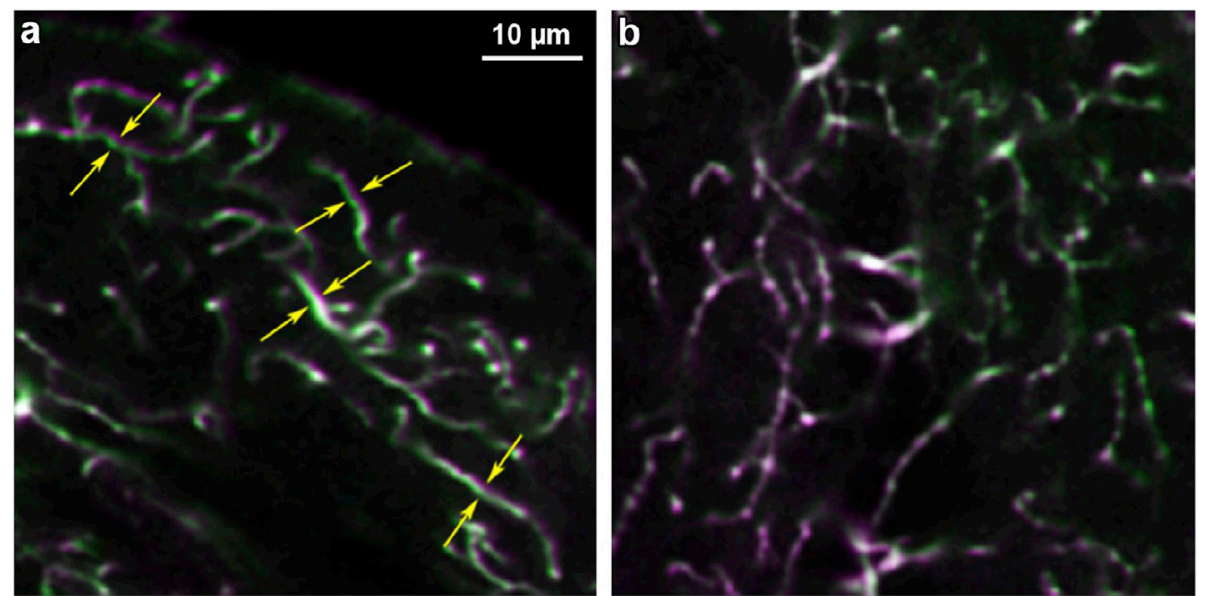

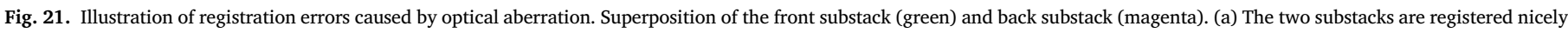

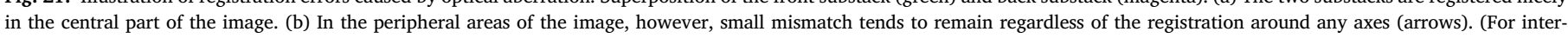
pretation of the references to colour in this figure legend, the reader is referred to the web version of this article.)

problem; although long-wavelength infrared light of the excitation laser is less prone to diffraction and scattering, emission light of shortwavelength from the labeled signals suffers from the same optical problem as in single-photon confocal microscopy. A promising alternative solution to obtain a thick high-resolution image stack is to stitch multiple substacks that are taken either from both sides of the specimen by sample flipping or from the same side by cutting off the sample surface progressively with microtomes. This alternative solution raises the problem of registration and stitching of partially overlapping 3D stacks. In this article, we proposed 2D-SIFT-in-3D-Space as an efficient method to address the registration and stitching of a variety of 3D image stacks. It specially allows tight adjustment of stacks of thin structures such as 
neurons to avoid seams between stitched substacks, or tiles, reducing errors in further 3D image processing and analyses. The registration is realized with an iterative combination of existing $2 \mathrm{D}$ local features in the 3D space. 2D-SIFT-in-3D-Space algorithm is not limited to neuron images of front and back substacks; it can be used on a variety of different datasets that require precise registration (See Figs. S2 and S3 and section 4 of the Supplementary data for additional examples). Also to help advanced analyses of 3D image stack in laser-scanning microscopy and to improve SIFT registration of dark samples, we provide a tool that improves intensity and contrast of serial section image stacks to compensate the loss induced by photobleaching and attenuation of the fluorescence (see Figs. S4 and S5 of the Supplementary data). Quantitative validation of 2D-SIFT-in-3D-Space is realized with the help of an in silico virtual test data specially created for this study to simulate the noise in fluorescent laser-scanning microscopy. Given the huge literature and software libraries dedicated to image registration, other programs, as discussed in the related work section, would in principle have the potential to also perform the registration problem illustrated here on Drosophila brain. Different image datasets possess diverse characteristic features that affect registration, and each registration method has to be tuned properly for each purpose for a fair comparison. A quantitative comparison with all registration solutions and exhaustive investigation of the whole parameter spaces of each of all these solutions would be an important milestone. Such an exhaustive work has recently been proposed for instance for particle tracking [45]. To target a similar milestone for 3D image registration of fluorescent microscopy, annotated image datasets with ground-truth registration solution would be required. Manual annotation and registration of such datasets would be very difficult and time consuming. The simulator proposed in our manuscript therefore opens the way to the quantitative benchmark of various registration methods of 3D images acquired with fluorescent microscopy.

Other interesting perspectives include evolution of our algorithm. We considered situations where it was possible to assume that the samples were not deforming during two imaging steps. In this framework, we logically considered rigid registration. In case where this assumption would not hold it would then be necessary to realize non rigid transformation. Also, the proposed algorithm allow to register multiple channels in fluorescence stack of images. However, the registration is based on one single channel (the best contrasted one), and the computed transformation is then applied to the other channels. SIFT can be applied to multiple component 3D images in principle and a joint registration of the channel could be considered as a further improvement.

\section{Information sharing statement}

The described algorithm 2D-SIFT-in-3D-Space Volume Stitching is implemented using the Java programming language and provided as Open Source plugin module of Fiji/ImageJ. It depends in Fiji on mpicbg package for SIFT and RANSAC and stitching package to fuse image stacks. The source code of the algorithm is available via Github (https:// github.com/rosahuaman/2D-SIFT-in-3D-Space-master). The simulator of laser-scanning microscopy image stacks was performed using Matlab (Natick, Massachusetts). The script code and artificially generated datasets from simulation are available via https://www.creatis.insa-lyon.fr/ site7/fr/MicroTools.

\section{Conflict of interest}

Non declared.

\section{Acknowledgements}

We thank Hanchuan Peng and Henrik Skibbe for their comments and suggestions, and Haruka Hirose, Youko Ishida, Ryoko Tatsumi, Jun Tanimura, Tomoko Yano, Takaaki Miyazaki and Asako Tsubouchi for their technical and scientific assistance for sample data preparation. We thank Bloomington Drosophila Stock Centre for fly strains and Hybridoma Bank for antibodies. This study is supported by the Strategic Research Program for Brain Sciences and CREST Program by the Japan Science and Technology Agency (JST)/Japan Agency for Medical Research and Development (AMED) to KI. The authors thank ZEISS for the experiments on light sheet fluorescence microscopy.

\section{Appendix A. Supplementary data}

Supplementary data related to this article can be found at https://doi. org/10.1016/j.compbiomed.2017.10.027.

\section{References}

[1] A.H. Brand, N. Perrimon, Targeted gene expression as a means of altering cell fates and generating dominant phenotypes, Development 118 (2) (1993) 401-415.

[2] K.M. Brown, G. Barrionuevo, A.J. Canty, V. De Paola, J.A. Hirsch, G.S. Jefferis, J. Lu, M. Snippe, I. Sugihara, G.A. Ascoli, The diadem data sets: representative light microscopy images of neuronal morphology to advance automation of digital reconstructions, Neuroinformatics 9 (2-3) (2011) 143-157.

[3] C.-T. Shih, O. Sporns, S.-L. Yuan, T.-S. Su, Y.-J. Lin, C.-C. Chuang, T.-Y. Wang, C. C. Lo, R.J. Greenspan, A.-S. Chiang, Connectomics-based analysis of information flow in the Drosophila brain, Curr. Biol. 25 (10) (2015) 1249-1258.

[4] L. Fritzky, D. Lagunoff, Advanced methods in fluorescence microscopy, Anal. Cell. Pathol. 36 (1-2) (2013) 5-17.

[5] K. Ito, R. Okada, N.K. Tanaka, T. Awasaki, Cautionary observations on preparing and interpreting brain images using molecular biology-based staining techniques, Microsc. Res. Tech. 62 (2) (2003) 170-186.

[6] K. Chung, J. Wallace, S.-Y. Kim, S. Kalyanasundaram, A.S. Andalman, T.J. Davidson, J.J. Mirzabekov, K.A. Zalocusky, J. Mattis, A.K. Denisin, et al., Structural and molecular interrogation of intact biological systems, Nature 497 (7449) (2013) 332-337.

[7] H. Hama, H. Kurokawa, H. Kawano, R. Ando, T. Shimogori, H. Noda, K. Fukami, A. Sakaue-Sawano, A. Miyawaki, Scale: a chemical approach for fluorescence imaging and reconstruction of transparent mouse brain, Nat. Neurosci. 14 (11) (2011) 1481-1488.

[8] B.W. Graf, S.A. Boppart, Imaging and analysis of three-dimensional cell culture models, Live Cell Imaging Methods Protoc. (2010) 211-227.

[9] T. Ragan, L.R. Kadiri, K.U. Venkataraju, K. Bahlmann, J. Sutin, J. Taranda, I. Arganda-Carreras, Y. Kim, H.S. Seung, P. Osten, Serial two-photon tomography for automated ex vivo mouse brain imaging, Nat. Methods 9 (3) (2012) 255-258.

[10] T. Zheng, Z. Yang, A. Li, X. Lv, Z. Zhou, X. Wang, X. Qi, S. Li, Q. Luo, H. Gong, et al., Visualization of brain circuits using two-photon fluorescence micro-optical sectioning tomography, Opt. Express 21 (8) (2013) 9839-9850.

[11] A. Cardona, Towards semi-automatic reconstruction of neural circuits, Neuroinformatics 11 (1) (2013) 31-33.

[12] E. Meijering, Neuron tracing in perspective, Cytom. Part A 77 (7) (2010) 693-704.

[13] B. Zitova, J. Flusser, Image registration methods: a survey, Image Vis. Comput. 21 (11) (2003) 977-1000.

[14] I.N. Figueiredo, S. Moura, J.S. Neves, L. Pinto, S. Kumar, C.M. Oliveira, J.D. Ramos, Automated retina identification based on multiscale elastic registration, Comput. Biol. Med. 79 (2016) 130-143.

[15] M. Yang, H. Ding, L. Zhu, G. Wang, Ultrasound fusion image error correction using subject-specific liver motion model and automatic image registration, Comput. Biol. Med. 79 (2016) 99-109.

[16] Y. Song, H. Lee, H.C. Kang, J. Shin, G.-S. Hong, S.H. Park, J. Lee, Y.-G. Shin, Interactive registration between supine and prone scans in computed tomography colonography using band-height images, Comput. Biol. Med. 80 (2017) 124-136.

[17] J. Kim, J. Lee, J.W. Chung, Y.-G. Shin, Locally adaptive 2D-3D registration using vascular structure model for liver catheterization, Comput. Biol. Med. 70 (2016) 119-130.

[18] A.D. Savva, T.L. Economopoulos, G.K. Matsopoulos, Geometry-based vs. intensitybased medical image registration: a comparative study on 3D CT data, Comput. Biol. Med. 69 (2016) 120-133.

[19] T. Rohlfing, User Guide to the Computational Morphometry Toolkit, Neuroscience Program, SRI International, Menlo Park, CA, 2011.

[20] S. Preibisch, S. Saalfeld, P. Tomancak, Globally optimal stitching of tiled 3D microscopic image acquisitions, Bioinformatics 25 (11) (2009) 1463-1465.

[21] M.N. Economo, N.G. Clack, L.D. Lavis, C.R. Gerfen, K. Svoboda, E.W. Myers, J. Chandrashekar, A platform for brain-wide imaging and reconstruction of individual neurons, Elife 5 (2016), e10566.

[22] S. Preibisch, S. Saalfeld, J. Schindelin, P. Tomancak, Software for bead-based registration of selective plane illumination microscopy data, Nat. Methods 7 (6) (2010) 418-419.

[23] M.N. Economo, N.G. Clack, B.J. Arthur, C. Bruns, E. Bas, J. Chandrashekar, Registration and resampling of large-scale 3D mosaic images, in: Proceedings of BioImage Informatics Conference, 2015.

[24] J. Li, N.M. Allinson, A comprehensive review of current local features for computer vision, Neurocomputing 71 (10) (2008) 1771-1787.

[25] D.G. Lowe, Distinctive image features from scale-invariant keypoints, Int. J. Comput. Vis. 60 (2) (2004) 91-110. 
[26] P. Scovanner, S. Ali, M. Shah, A 3-dimensional SIFT descriptor and its application to action recognition, in: Proceedings of the 15th ACM International Conference on Multimedia, ACM, 2007, pp. 357-360.

[27] G.T. Flitton, T.P. Breckon, N.M. Bouallagu, Object recognition using 3D SIFT in complex ct volumes, in: BMVC, 2010, pp. 1-12.

[28] W. Cheung, G. Hamarneh, N-sift: N-dimensional scale invariant feature transform for matching medical images, in: Biomedical Imaging: from Nano to Macro, 2007, IEEE, 2007, pp. 720-723. ISBI 2007. 4th IEEE International Symposium on.

[29] C. Paganelli, M. Peroni, F. Pennati, G. Baroni, P. Summers, M. Bellomi, M. Riboldi, Scale invariant feature transform as feature tracking method in $4 \mathrm{~d}$ imaging: a feasibility study, in: Engineering in Medicine and Biology Society (EMBC), IEEE, 2012, pp. 6543-6546, 2012 Annual International Conference of the IEEE.

[30] S. Allaire, J.J. Kim, S.L. Breen, D.A. Jaffray, V. Pekar, Full orientation invariance and improved feature selectivity of $3 \mathrm{~d}$ sift with application to medical image analysis, in: Computer Vision and Pattern Recognition Workshops, 2008, IEEE, 2008, pp. 1-8. CVPRW'08. IEEE Computer Society Conference on.

[31] D. Ni, Y. Qu, X. Yang, Y.P. Chui, T.-T. Wong, S.S. Ho, P.A. Heng, Volumetric ultrasound panorama based on 3d sift, in: International Conference on Medical Image Computing and Computer-assisted Intervention, Springer, 2008, pp. 52-60.

[32] K. Ito, K. Shinomiya, M. Ito, J.D. Armstrong, G. Boyan, V. Hartenstein, S. Harzsch, M. Heisenberg, U. Homberg, A. Jenett, et al., A systematic nomenclature for the insect brain, Neuron 81 (4) (2014) 755-765.

[33] S.-y. Takemura, A. Bharioke, Z. Lu, A. Nern, S. Vitaladevuni, P.K. Rivlin, W.T. Katz, D.J. Olbris, S.M. Plaza, P. Winston, et al., A visual motion detection circuit suggested by Drosophila connectomics, Nature 500 (7461) (2013) 175-181.

[34] S. Busch, M. Selcho, K. Ito, H. Tanimoto, A map of octopaminergic neurons in the Drosophila brain, J. Comp. Neurol. 513 (6) (2009) 643-667.
[35] K.E. White, D.M. Humphrey, F. Hirth, The dopaminergic system in the aging brain of Drosophila, Front. Neurosci. 4 (2010) 205.

[36] T. Lindeberg, Feature detection with automatic scale selection, Int. J. Comput. Vis. 30 (2) (1998) 79-116.

[37] S. Saalfeld, P. Tomancák, Automatic landmark correspondence detection for imagej, in: Proceedings of the ImageJ User and Developer Conference, 2008, pp. 128-133.

[38] S. Saalfeld, A. Cardona, V. Hartenstein, P. Tomančák, As-rigid-as-possible mosaicking and serial section registration of large sstem datasets, Bioinformatics 26 (12) (2010) i57-i63.

[39] G. Herberich, R. Windoffer, R. Leube, T. Aach, Signal and noise modeling in confocal laser scanning fluorescence microscopy, in: Medical Image Computing and Computer-Assisted Intervention-MICCAI 2012, 2012, pp. 381-388.

[40] C.H. Li, C. Lee, Minimum cross entropy thresholding, Pattern Recognit. 26 (4) (1993) 617-625.

[41] S. Preibisch, F. Amat, E. Stamataki, M. Sarov, R.H. Singer, E. Myers, P. Tomancak, Efficient bayesian-based multiview deconvolution, Nat. Methods 11 (6) (2014) 645-648.

[42] S. Saalfeld, R. Fetter, A. Cardona, P. Tomancak, Elastic volume reconstruction from series of ultra-thin microscopy sections, Nat. Methods 9 (7) (2012) 717-720.

[43] C.J. Sheppard, M. Gu, Aberration compensation in confocal microscopy, Appl. Opt. 30 (25) (1991) 3563-3568.

[44] M.J. Booth, M.A. Neil, R. Juškaitis, T. Wilson, Adaptive aberration correction in a confocal microscope, Proc. Natl. Acad. Sci. 99 (9) (2002) 5788-5792.

[45] N. Chenouard, I. Smal, F. De Chaumont, M. Maška, I.F. Sbalzarini, Y. Gong, J. Cardinale, C. Carthel, S. Coraluppi, M. Winter, et al., Objective comparison of particle tracking methods, Nat. Methods 11 (3) (2014) 281-289. 